\title{
Analysis of Inertial Migration of Neutrally Buoyant Particle Suspensions in a Planar Poiseuille Flow with a Coupled Lattice Boltzmann Method-Discrete Element Method
}

\author{
Wenwei Liu (刘文巍), Chuan-Yu Wu (邬传宇)* \\ Department of Chemical and Process Engineering, University of Surrey, \\ Guildford, GU2 7XH, UK
}

\begin{abstract}
In this study a hybrid numerical framework for modelling solid-liquid multiphase flow is established with a single-relaxation-time lattice Boltzmann method and the discrete element method implemented with the Hertz contact theory. The numerical framework is then employed to systematically explore the effect of particle concentration on the inertial migration of neutrally buoyant particle suspensions in planar Poiseuille flow. The results show that the influence of particle concentration on the migration is primarily determined by the characteristic channel Reynolds number $R e_{0}$. For relatively low $\operatorname{Re}_{0}\left(\operatorname{Re}_{0}<20\right)$, the migration behaviour can only be observed at a very low particle concentration $(\leq 5 \%)$. However, when $R e_{0}>20$ the migration behaviour can be observed at a high concentration $(\geq 20 \%)$. Furthermore, a focusing number $F_{c}$ is proposed to characterise the degree of inertial migration. It was found that the inertial migration can be classified into three regimes depending on two critical values of the focusing number, $F_{c}{ }^{+}$and $F_{c}^{-}: i$ ) when $F_{c}>F c^{+}$, a full inertial migration occurs; $\left.i i\right)$ when $F_{c}<F c^{-}$, particles are laterally unfocused; iii) when $F c^{-}<F_{c}<F c^{+}$, a partially inertial migration takes place.
\end{abstract}

Keywords: Lattice Boltzmann method; Discrete element method; Inertial migration; Pipe flow 


\section{1. Introduction}

2 The transport of solid particles carried by liquid in pipes and vessels is a complex problem,

3 which ubiquitously exists in nature and industrial processes, such as chemical production, food

4 processing, pharmaceutics, oil and mining engineering. Various studies have shown that the

5 behaviour for rigid spheres in fluid flow depends strongly on the specific bulk flow geometry

6 and on whether or not the particle is neutrally buoyant [1]. Neutrally buoyant particles are the

7 particles with the same density as the immersing fluid, implying that particles are suspended in

8 the fluid. For a simple Poiseuille flow with a dilute suspension of neutrally buoyant spheres,

9 Segré and Silberberg [2,3] first observed that a single rigid sphere in pipe flow migrated to an

10 equilibrium position with its center located around $0.6 R$, with $R$ being the pipe radius. The

11 phenomenon of radial migration driven by inertia was termed as the tubular pinch effect (or

12 Segré-Silberberg effect), implying that the uniform distribution of particles over the pipe crosssection converges, or is 'pinched', to a narrow annulus as the suspension flows. These observations prompted a strong interest in the suspension community to identify the underlying mechanism and provide a theoretical explanation of this interesting phenomenon [1,4-31]. $[1,4,5]$. For example, Ho et al. [1] theoretically analysed the lateral migration of a neutrally buoyant rigid sphere in both simple shear flow and Poiseuille flow in $2 \mathrm{D}$ and confirmed the Segré-Silberberg effect in the 2D Poiseuille flow. But for the simple shear flow, the equilibrium position was found to be at the center line between the walls. The lateral forces and particle trajectories were also calculated based on the theoretical solutions. Schonberg and Hinch [4] 
Reynolds number of order unity, by means of a singular perturbation expansion with an Oseenlike region. Assuming that the sphere is sufficiently small, the disturbance of the background flow is negligible. Thus, the convective terms were linearized and analytical solutions were derived. Their results showed that the equilibrium position moves towards the wall as the Reynolds number increases and the migration velocity increases more slowly than quadratically. Asmolov [5] calculated the lift force on both neutrally and non-neutrally buoyant small spheres in a channel flow at a large Reynolds number using the matched asymptotic expansion. The results indicated that the wall-induced inertia is significant in the thin layers near the walls, while the wall effect can be neglected in the major portion of the flow excluding near-wall layers. the wall as $R e$ increases. Long-lived trains of particles aligned with the flow were also observed.

44 Furthermore, along with the matched asymptotic expansion method, the lateral migration force 
exerted on a rigid neutrally buoyant sphere in a Poiseuille flow was evaluated and discussed [10]. The theories showed that three potential sources of lift forces could lead to the tubular pinch effect, including the Saffman force, the wall lubrication repulsion, and the lift force caused by the curvature of the velocity profile in a Poiseuille flow. Choi and Lee [11] carried out a 3D holographic analysis of the inertial migration of spherical particles in micro-scale pipe flows with the Reynolds number $1.6 \leq R e \leq 77.4$ and the pipe-to-particle size ratio $D / d=50,23$, 12 , and found that the micro-scale flows share the similar migration behaviour to that in the macro-scale flows. Di Carlo et al. [12,13] reviewed the inertial migration of particles in microfluidics and summarized the key factors in controlling the micro-flow, which shed light on the potential application of inertial migration in enhanced mixing, particle separation, and bioparticle focusing. Seo et al. [14-15] investigated particle migration and single-line particle focusing in both micro-scale pipe and square channel flows using a holographic technique. The effects of blockage ratio, flow rate, and entry length on particle migration induced by fluid elasticity were evaluated. A dimensionless focusing number was also proposed to describe the focusing behaviour of particles.

Computer simulation has become a powerful tool in analyzing particle migration in pipe flows. The complex nature of hydrodynamic interactions between the particles and the liquid gives rise to great challenges in predicting the behaviours of both the particles and the liquid flow, which can be fulfilled by numerical modeling to fully understand the behaviour of particle-liquid flows. The numerical techniques vary from the direct numerical simulation (DNS) [18], the finite element method (FEM) [19], computational fluid dynamics (CFD) [20,21] to the fictitious domain method (FDM) [22] and the lattice Boltzmann method (LBM) [23,24]. 
With these numerical approaches, comprehensive studies on the migration of particle suspensions were carried out in both $2 \mathrm{D}$ and $3 \mathrm{D}$, where details of the velocity profiles, the lift forces, the equilibrium positions were provided. Moreover, the influences of Reynolds numbers, pipe-particle size ratios and pipe lengths can all be obtained and analysed [25-32].

Among these numerical techniques, LBM has been widely applied in the simulation of solid-liquid suspensions due to its simplicity in parallel programing, flexibility in handling the

boundary conditions, high space-time resolution and greatly reduced computational time. Inamuro et al. [25] simulated the motions of a single and two lines of neutrally buoyant circular cylinders in fluid between two flat parallel walls with the Reynolds number of $12<\operatorname{Re}<96$, and the channel-cylinder ratio of $2 \leq D / d \leq 4$. Chun and Ladd [26] numerically modelled inertial migration of neutrally buoyant particles in a $3 \mathrm{D}$ square duct with $100<\operatorname{Re}<1,000$, where multiple equilibrium positions located near a corner or at the center of an edge were reported. Yan and co-workers $[27,28]$ discussed the hydrodynamic interactions of two solid bodies in 2D confined linear shear flow at a finite Reynolds number. Furthermore, they analysed the transport and sedimentation of suspended particles in a 3D square duct with particle concentration $0.13<\Phi<0.34$, which covers a wide range of buoyancy and Reynolds numbers. Chun et al. [29] modelled the migration of particle suspensions in combined plane CouettePoiseuille flows with a particle volume fraction $\Phi=0.4$ and a channel-particle size ratio $D / d=44.3,22.3$. The behaviours of migration are classified into three groups based on the value of a characteristic force, i.e. the relative magnitude of the body force against the wall-driving force. Sun and co-workers [30,31] applied an immersed boundary lattice Boltzmann method (IB-LBM) with multi relaxation time (MRT)-LB equation to investigate the hydrodynamic 
focusing of particles in straight channel flows. A particle focusing entropy was proposed to quantitatively characterise the focusing behaviour.

It is clear that previous studies on solid-liquid pipe flows were mostly confined to dilute suspensions with a very low particle concentration $(<1 \%)$. Much effort was taken to reveal the mechanism of the Segré-Silberberg effect both theoretically and experimentally. The balance between the lift forces and the wall repulsion determines the equilibrium position of the migration, which moves towards the wall with the increase of the channel Reynolds number. However, little attention has been paid to the migration behaviour when the particle concentration increases. It is still unclear how the particle solid fraction will affect the inertial migration of particle suspension and whether there is a critical solid fraction governing the migration behaviour. Therefore, the aim of this study is to explore the impact of particle concentration on the inertial migration of particle suspensions. For this purpose, we first develop a numerical simulation framework using a lattice Boltzmann method (LBM) coupled with a discrete element method (DEM) that is implemented with the Hertzian contact model. The developed model is then validated using the data reported in literature. We then perform a systematical study on the migration of neutrally buoyant particles in a planar Poiseuille flow with particle concentration ranging from $1 \%$ to $50 \%$ and the channel Reynolds number varying between 4 and 100. The effects of the particle concentration and Reynolds number are fully analysed and discussed. 
108 2. Numerical model

\subsection{Lattice Boltzmann method (LBM)}

LBM originated from the lattice gas automata (LGA) method, which can be considered as a simplified fictitious molecular dynamics model to simulate fluid flows. In LBM, the fluid domain is discretised with a number of lattices, which is similar to the mesh grid of macroscopic computational fluid dynamics. In each lattice, the packets of fluid are described by the density distribution functions $f_{i}(\mathbf{x}, \mathbf{t})$, which relate the probable amount of the fictitious fluid 'particles' moving with a discrete speed in a discrete direction at each lattice node within each time increment. The density distribution functions are analogous to the continuous, microscopic density function of the Boltzmann equation. In every lattice, the density distribution functions follow the same discretised speed model, for which various algorithms are proposed. In our numerical framework, the widely used discretization schemes, i.e. the D2Q9 model in 2D and the D3Q19 model in 3D, are adopted, respectively. In the D2Q9 model, the fluid particles at each lattice are allowed to move to its 8 immediate neighbours with 8 different velocities, $\mathbf{e}_{i}(i=1 \sim 8)$, while the particles move to 26 neighbours with 18 discretised velocities in D3Q19 model, as shown in Fig. 1. Similarly, there are also other discretised models, such as D2Q7 model in 2D, D3Q15 and D3Q27 models for 3D cases. 

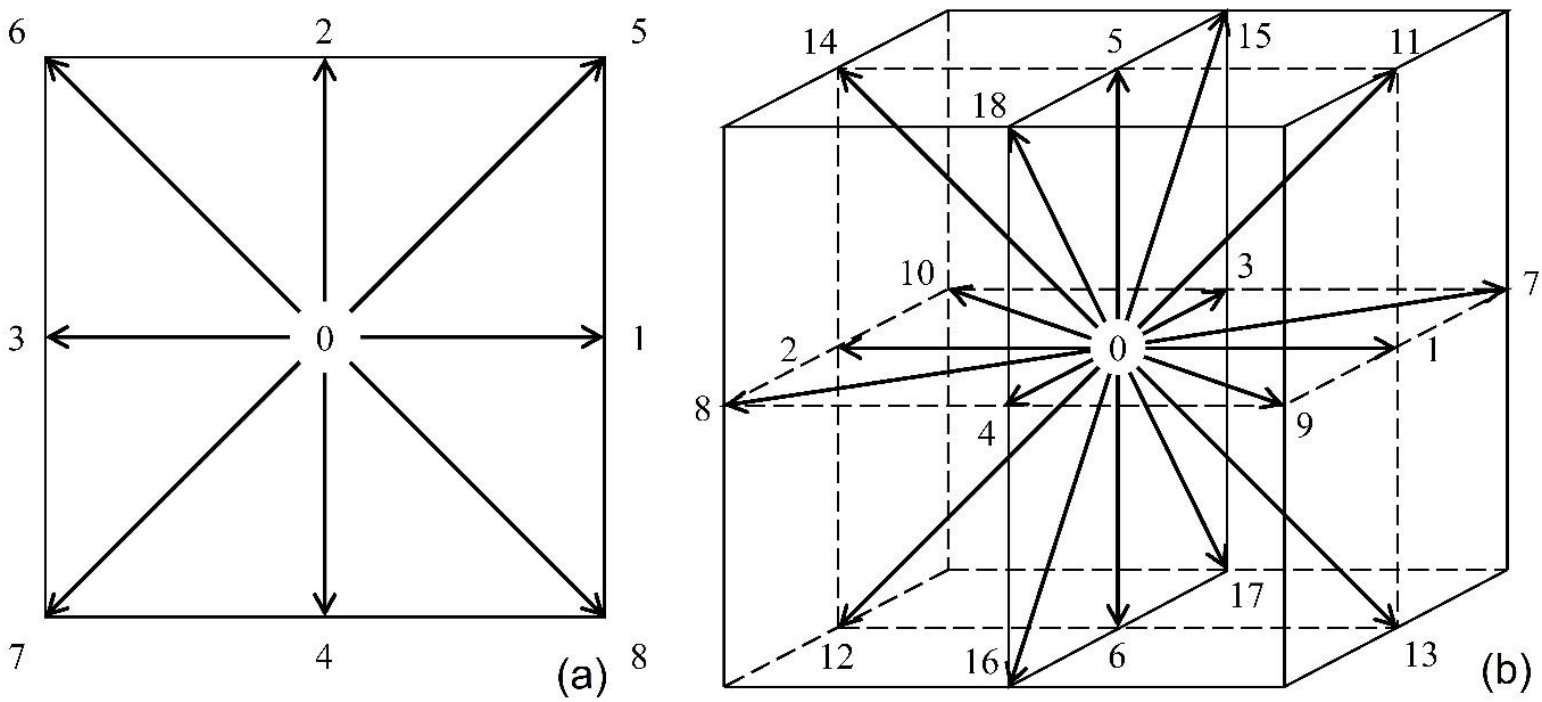

Fig.1 The (a) D2Q9 and (b) D3Q19 lattice speed model.

The density distribution functions $f_{i}(\mathbf{x}, \mathbf{t})$ are governed by the lattice Boltzmann equation

$$
f_{i}\left(\mathbf{x}+\mathbf{e}_{i} \Delta t, t+\Delta t\right)=f_{i}(\mathbf{x}, t)+\Omega_{i}\left[f_{i}(\mathbf{x}, t)\right]+F_{i} \Delta t,
$$

where the vector $\mathbf{x}$ denotes the position of the node for which the calculation is being carried out, $\Delta t$ is the explicit time step, $F_{i}$ represents a body force acting on the fluid and $\Omega_{i}\left[f_{i}(\mathbf{x}, \mathrm{t})\right]$ is the collision operator that controls the relaxation rate of the density distribution functions.

At each node, Eq. (1) is evolved by the collision and streaming processes at each time step.

Collision (also known as relaxation) redistributes the functions that arrive at each node and then streaming (also known as convection) propagates the redistributed functions to their nearest neighbour nodes. Over a number of time steps the ordering of the streaming and collision operations is irrelevant. For the collision process, a single-relaxation-time LBE 
141 linearized by the Bhatnagar-Gross-Krook approximation is employed [34-37], where the collision operator is given as

$$
\Omega_{i}=-\frac{\Delta t}{\tau}\left[f_{i}(\mathbf{x}, t)-f_{i}^{e q}(\mathbf{x}, t)\right]
$$

Here, $\tau$ is the dimensionless relaxation parameter and $f_{i}^{e q}(\mathbf{x}, \mathrm{t})$ is the equilibrium distribution

function defined as

$$
f_{i}^{e q}=\rho \omega_{i}\left[1+\frac{\mathbf{e}_{i} \cdot \mathbf{u}}{c_{s}^{2}}+\frac{\left(\mathbf{e}_{i} \cdot \mathbf{u}\right)^{2}}{2 c_{s}^{2}}-\frac{u^{2}}{2 c_{s}^{2}}\right]
$$

In Eq. (3), $\omega_{i}$ is the weight coefficient defined by the lattice speed model. For example, for the D2Q9 model, $\omega_{0}=4 / 9, \omega_{1,2,3,4}=1 / 9, \omega_{5,6,7,8}=1 / 36 . c_{s}=c / \sqrt{3}$ is the lattice sound speed, where $c=|\Delta x / \Delta t|$ is the lattice speed. $\rho$ and $\mathbf{u}$ are the macroscopic fluid density and velocity, which are determined as

$$
\begin{aligned}
& \rho=\sum_{i} f_{i}, \\
& \rho \mathbf{u}=\sum_{i} f_{i} \mathbf{e}_{i},
\end{aligned}
$$

and the fluid pressure field $p$ is determined by $p=c_{s}^{2} \rho$. To recover the incompressible Navier-

Stokes equations, the kinematic viscosity of the fluid $v$ is not directly used in the LBM model 


$$
v=\frac{1}{3}\left(\tau-\frac{1}{2}\right) \frac{(\Delta x)^{2}}{\Delta t}
$$

Based on Guo et al.'s work [38], in order to recover the correct form of the Navier-Stokes equation with a body force, the body force term $F_{i}$ in Eq. (1) should be expressed as

$$
F_{i}=\left(1-\frac{1}{2 \tau}\right) \omega_{i}\left[\frac{\mathbf{e}_{i}-\mathbf{u}}{c_{s}^{2}}+\frac{\left(\mathbf{e}_{i} \cdot \mathbf{u}\right)}{c_{s}^{4}} \mathbf{e}_{i}\right] \cdot \mathbf{F}
$$

where $\mathbf{F}$ is macroscopic body force. Correspondingly, the macroscopic fluid velocity in Eq. (4) should be modified as

$$
\rho \mathbf{u}=\sum_{i} f_{i} \mathbf{e}_{i}+\frac{\Delta t}{2} \mathbf{F}
$$

The recovery of the continuous Navier-Stokes equation from the lattice Boltzmann scheme can be obtained through a separation of scales by means of a Chapman-Enskog expansion analysis in power of the Knudsen number [33]. In order to reach their hydrodynamic limit, some symmetry properties must be satisfied for the particle density functions [39], which are not necessarily fulfilled in the conventional LBM numerical simulations. A more accurate and stable LBM with a regularization procedure before the collision step is available [39-40]. However, the numerical deviation in the current study due to the lack of the regularization can be negligible as the Knudsen number is very small [41]. For fluid flows of high Knudsen number, higher-order terms in the Chapman-Enskog expansion must be carefully considered. 


\subsection{Boundary conditions}

As velocity and pressure are not the primary variables in the LB formule, the normal pressure, velocity and their combined boundary conditions cannot be imposed directly, but alternative approaches to specify the boundary conditions in terms of the density distribution functions need to be developed [42]. In the current study, the 'no-slip' wall boundary conditions and the periodic boundary conditions are considered.

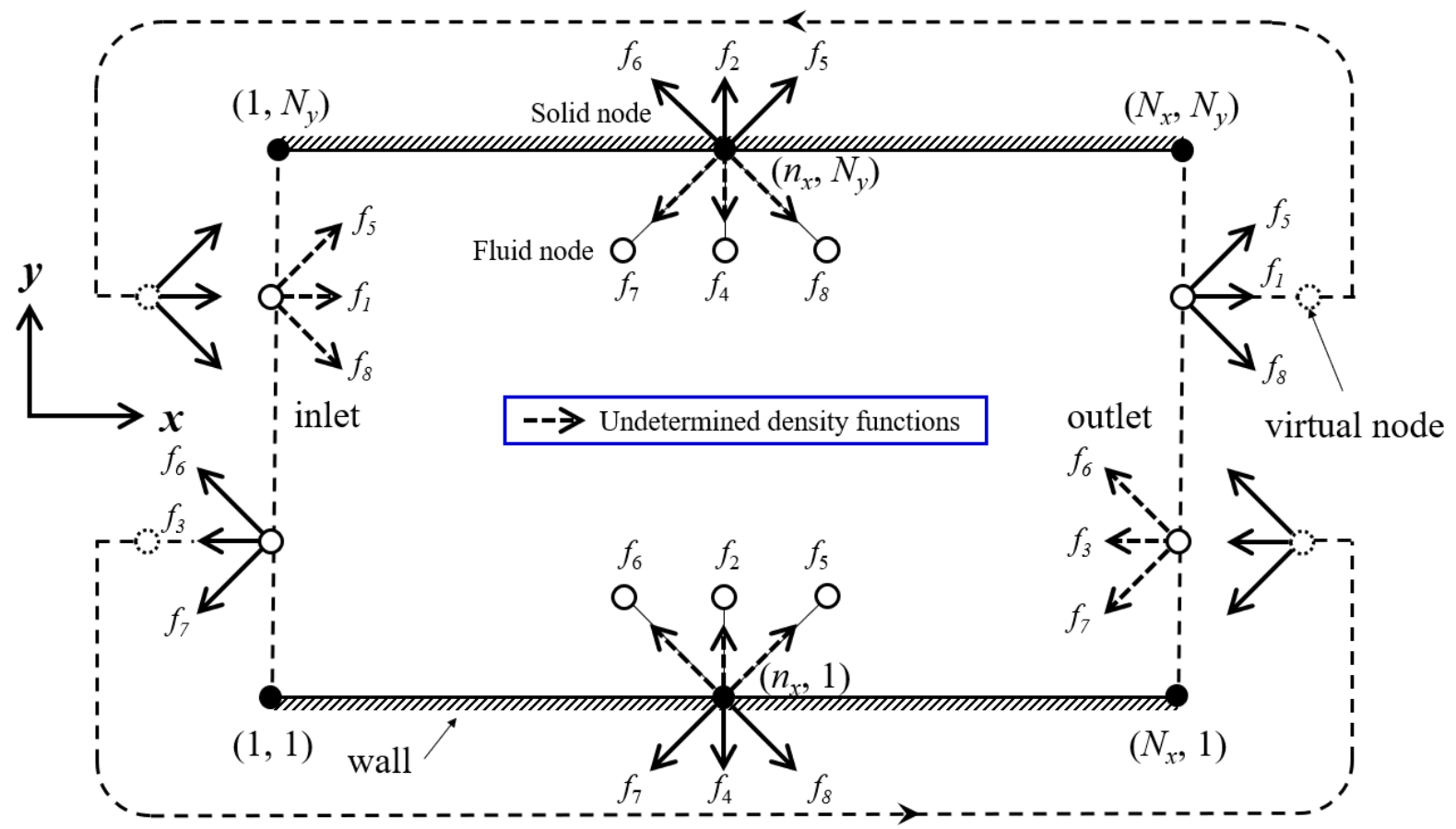
circles represent solid boundary nodes, while the open circles are the internal fluid nodes. 
wall can be imposed by the so-called bounce-back rule $[23,24]$. Suppose that a solid wall is defined by a set of grid nodes, as shown in Fig. 2. The nodes lying within the wall region are called solid nodes and the nodes in the fluid region are fluid nodes. If $i$ is a link (or direction) between a fluid node and a solid node, the bounce-back rule requires that the incoming fluid particles from the fluid node are reflected back to the node that it comes from, i.e.

$$
f_{-i}(\mathbf{x}, t+\Delta t)=f_{i}^{+}(\mathbf{x}, t)
$$

where the subscript $-i$ denotes the opposite direction of $i$ and $f_{i}^{+}$represents the post-collision density distribution function. Take the simple channel displayed in Fig. 2 as an example. The number of lattice nodes in the $x$ and $y$ directions are $N_{x}$ and $N_{y}$, respectively. In this case, the bounce-back rule simply gives $f_{4,7,8}\left(n_{x}, N_{y}\right)=f_{2,5,6}\left(n_{x}, N_{y}\right)$ at every node along the top wall and $f_{2,5,6}\left(n_{x}, 1\right)=f_{4,7,8}\left(n_{x}, 1\right)$ at the bottom wall, where $n_{x}$ denotes the $x$-coordinate of the solid node. This simple rule ensures that no tangential velocity exists along the fluid-wall interface, so that a 'no-slip' condition is imposed. Note that the collision and streaming processes are also carried out at the solid nodes, which has been proved to achieve a second-order accuracy [43]. This bounce-back rule works reasonably well for stationary walls and can also be extended to any shaped wall or obstacle (including stationary particles) in fluid flows.

Periodic boundary conditions are also implemented in the LBM. Distribution functions exiting the domain at one end are duplicated to a virtual node and transferred to the other end. A normal streaming process then takes place, where the distribution functions of the virtual node are treated as the input to the corresponding nodes at the other end. As shown in Fig. 2, 
the periodic boundary conditions for the D2Q9 model give $f_{1,5,8}\left(1, n_{y}\right)=f_{1,5,8}\left(N_{x}, n_{y}\right)$ at the inlet and $f_{3,6,7}\left(N_{x}, n_{y}\right)=f_{3,6,7}\left(1, n_{y}\right)$ at the outlet, where $n_{y}$ is the $y$-coordinate of the node at the periodic

221 boundary.

It should be noted that in our LBM, the solid particles that are immersed in the fluid are treated as moving wall boundaries and the interactions between the moving particles and the fluid are the most important part in the coupling of LBM and DEM. For the moving boundaries between the solid particles and the fluid, a physically correct 'no-slip' boundary condition is required to impose at the interface, i.e. the fluid adjacent to the particle surface should have identical velocity to that on the particle surface. The first step is to represent the particle using the lattice nodes. Figure 3 illustrates lattice discretization of a circular particle, where nodes interior and exterior to the particle are the solid and fluid nodes, respectively. These nodes are further classified into three categories: (1) fluid boundary node - a fluid node connected at least with one solid node; (2) solid boundary node - a solid node connected at least with one fluid node; and (3) interior solid node - a solid node not connected to any fluid node. A link between a fluid boundary node and a solid boundary node is called a boundary link. The surface of a solid particle is assumed to be located in the middle of the boundary links. Clearly, the stepwise lattice representation of the surface of a circular particle is neither accurate nor smooth unless a universal approach for any shaped particles. 


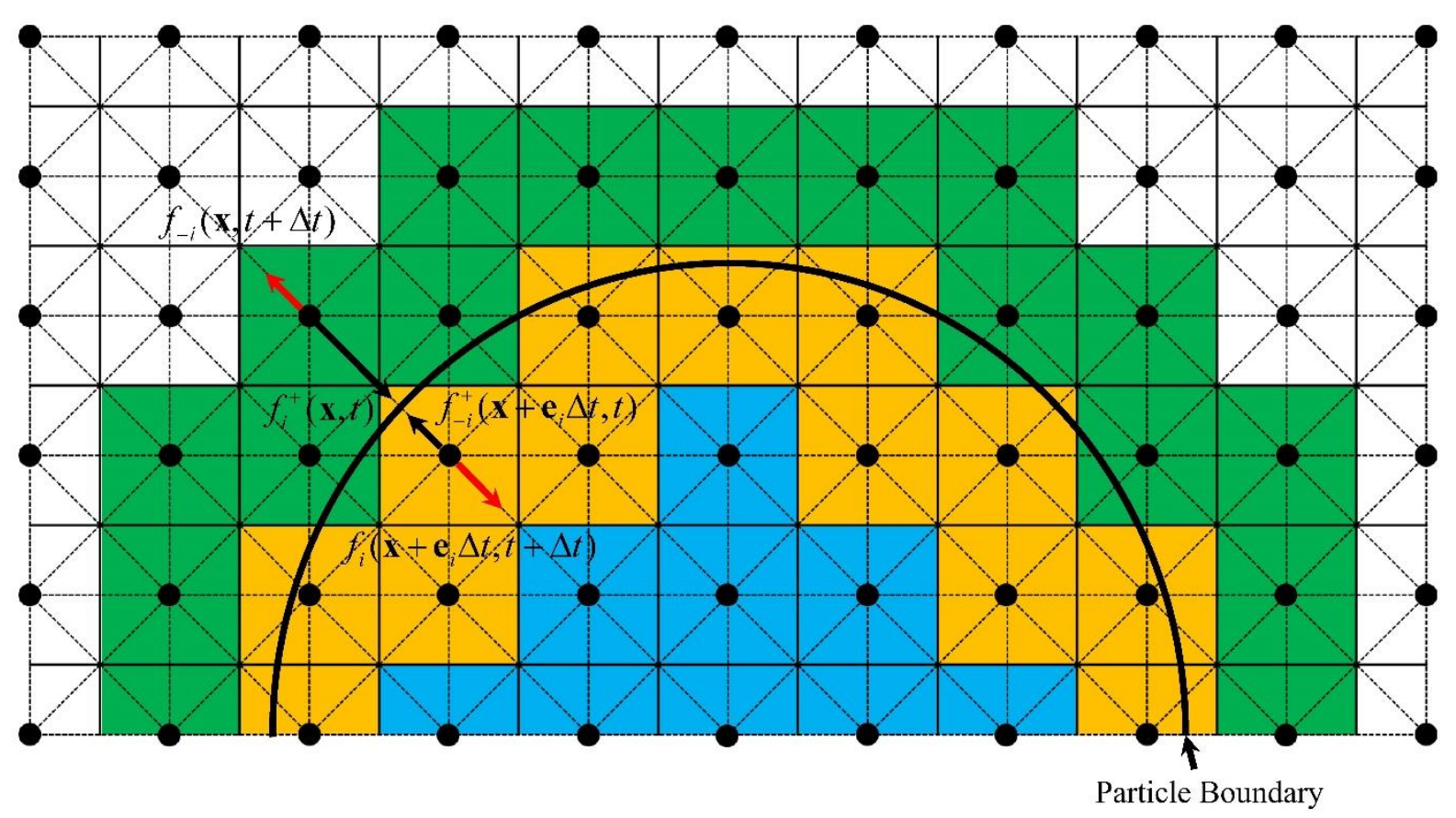

Fig. 3 Lattice representation of a circular solid particle showing solid boundary nodes (orange), fluid boundary nodes (green) and internal solid nodes (blue).

Based on the lattice representation, a modified bounce-back (MBB) method is applied to handle the interaction the fluid regime with suspended particles. The MBB method was proposed by Ladd $[23,24]$ as an improvement on the simple bounce-back technique for modelling the fluid-particle interaction with moving boundaries. This method enforces that the bounce-back occurs at the solid-fluid interface at halfway between the fluid boundary nodes and the solid boundary nodes. As shown in Fig. 3, the velocity of the boundary at the approximated interface is calculated as

$$
\mathbf{v}_{b}=\mathbf{U}_{p}+\mathbf{\Omega}_{p} \times\left(\mathbf{x}+\frac{1}{2} \mathbf{e}_{i} \Delta t-\mathbf{X}_{p}\right)
$$


254 the solid particle, respectively. With the boundary velocity at the link intersection, the bounceback procedures at the fluid and solid boundary nodes are, respectively,

$$
\begin{aligned}
& f_{-i}(\mathbf{x}, t+\Delta t)=f_{i}^{+}(\mathbf{x}, t)-2 \omega_{i} \rho \mathbf{v}_{b} \cdot \mathbf{e}_{i}, \\
& f_{i}\left(\mathbf{x}+\mathbf{e}_{i} \Delta t, t+\Delta t\right)=f_{-i}^{+}\left(\mathbf{x}+\mathbf{e}_{i} \Delta t, t\right)+2 \omega_{i} \rho \mathbf{v}_{b} \cdot \mathbf{e}_{i} .
\end{aligned}
$$

Consequently, the force exerted on the solid particle as a result of the $\mathrm{BB}$ and momentum transfer is calculated from the net change at the links as follows

$$
\mathbf{F}_{f,-i}\left(\mathbf{x}+\frac{1}{2} \mathbf{e}_{i} \Delta t, t+\frac{1}{2} \Delta t\right)=2\left[f_{i}^{+}(\mathbf{x}, t)-f_{-i}^{+}\left(\mathbf{x}+\mathbf{e}_{i} \Delta t, t\right)-2 \omega_{i} \rho \mathbf{v}_{b} \cdot \mathbf{e}_{i}\right] \cdot \mathbf{e}_{i} .
$$

The total hydrodynamic force and the torque are then calculated by summing over all the boundary links, i.e.

$$
\mathbf{F}_{f}=\sum_{i} \mathbf{F}_{f,-i}
$$

$$
\mathbf{M}_{f}=\sum_{i}\left(\mathbf{x}+\frac{1}{2} \mathbf{e}_{i} \Delta t-\mathbf{X}_{p}\right) \times \mathbf{F}_{f,-i} .
$$

\subsection{Discrete element method}




$$
m \frac{d \mathbf{U}_{p}}{d t}=\mathbf{F}_{f}+\mathbf{F}_{c}+\mathbf{G}
$$

$$
I \frac{d \mathbf{\Omega}_{p}}{d t}=\mathbf{M}_{f}+\mathbf{M}_{c}
$$

where $\mathbf{U}_{p}$ and $\boldsymbol{\Omega}_{p}$ are, respectively, the transitional velocity and the rotational velocity of an individual particle, which are the same as used in Eq. (9). $m$ is the particle mass and $I=2 / 5 m r_{p}{ }^{2}$ is the moment of inertia. $\mathbf{G}$ is the gravity. $\mathbf{F}$ and $\mathbf{M}$ denote the force and the torque acting on each individual particle, and the subscript $f$ and $c$ represent sources of these interactions, from the fluid and the interparticle collision, respectively. The collision force and the torque include

$$
\begin{aligned}
& \boldsymbol{F}_{c}=F_{n} \mathbf{n}+F_{s} \mathbf{t}_{s}, \\
& \boldsymbol{M}_{c}=r_{p} F_{s}\left(\mathbf{n} \times \mathbf{t}_{s}\right)+M_{r}\left(\mathbf{t}_{s} \times \mathbf{n}\right)+M_{t} \mathbf{n},
\end{aligned}
$$

where $F_{n}$ is the normal force including the elastic contact force and the damping force, $F_{s}$ is the tangential force due to the sliding friction, $M_{r}$ is the rolling resistance and $M_{t}$ is the twisting resistance. $r_{p}$ is the particle radius. $\mathbf{n}, \mathbf{t}_{s}$ and $\mathbf{t}_{r}$ are the normal, tangential and rolling direction unit vectors, respectively.

The normal force acts in the direction of the unit vector $\mathbf{n}$ that points parallel to the line connecting the centers of the two particles, denoted by $i$ and $j$, such that $\mathbf{n}=\left(\mathbf{x}_{j}-\mathbf{x}_{i}\right) /\left|\mathbf{x}_{j}-\mathbf{x}_{i}\right|$. We consider two particles with radii $r_{p, i}$ and $r_{p, j}$, elastic moduli $E_{i}$ and $E_{j}$, and Poisson's ratios $\sigma_{i}$ and $\sigma_{j}$. An effective particle radius $R$ and an effective elastic moduli $E$ are defined as 


$$
\frac{1}{R} \equiv \frac{1}{r_{i}}+\frac{1}{r_{j}}
$$

$$
\frac{1}{E} \equiv \frac{1-\sigma_{i}^{2}}{E_{i}}+\frac{1-\sigma_{j}^{2}}{E_{j}} \text {. }
$$

The particle normal overlap $\delta_{N}$ is defined by $\delta_{N}=r_{p, i}+r_{p, j}-\left|\mathbf{x}_{j}-\mathbf{x}_{i}\right|$, where $\mathbf{x}_{i}$ and $\mathbf{x}_{j}$ denote the particle centroid positions. The normal force is described by the Hertz contact model along with a damping term, and expressed as follows

$$
F_{n}=-k_{N} \delta_{N}^{1.5}-\eta_{N} \mathbf{v}_{R} \cdot \mathbf{n},
$$

where $k_{N}=\frac{4}{3} E \sqrt{R}$ is the normal stiffness, $\eta_{N}$ is the normal dissipation coefficient, and $\mathbf{v}_{R}$ is the relative velocity at the contact point. The contact radius $a$ is geometrically related to the particle radius and the normal overlap as $a=\sqrt{R \delta_{N}}$. direction of the "rolling" velocity. An expression for the rolling displacement of arbitraryshaped particles was derived by Bagi and Kuhn [46]. Applying the rate of this expression to spherical particles of equal sizes yields an equation for the "rolling velocity" $\mathbf{v}_{L}$ of particle $i$ as

$$
\mathbf{v}_{L}=-R\left(\mathbf{\Omega}_{i}-\mathbf{\Omega}_{j}\right) \times \mathbf{n}
$$

An expression for the rolling resistance torque $M_{r}$ is postulated in the form

$$
M_{r}=-k_{R} \xi_{R} \cdot \mathbf{t}_{r}-\eta_{R} \mathbf{v}_{L} \cdot \mathbf{t}_{r}
$$


313 where the direction of rolling is $\mathbf{t}_{r}=\mathbf{v}_{L} /\left|\mathbf{v}_{L}\right|$ and the rolling displacement is $\boldsymbol{\xi}_{R}=\int_{t_{0}}^{t} \mathbf{v}_{L}(\tau) \cdot \mathbf{t}_{r} d \tau$. $314 k_{R}$ is the rolling stiffness and $\eta_{R}$ is the rolling damping coefficient. The first term in Eq. (18) 315 represents a spring force and the second term accounts for the effect of damping. If the 316 magnitude of rolling resistance torque $\left|M_{r}\right|$ is greater than a critical value $M_{r, c r i t}$, then it keeps constant as $M_{r}=-M_{r, \text { crit }}$.

The standard sliding model is proposed by Cundall and Strack [47], for which the tangential force $F_{S}$ is given by a linear spring-dashpot,

$$
F_{s}=-k_{T} \delta_{T} \cdot \mathbf{t}_{s}-\eta_{T} \mathbf{v}_{s} \cdot \mathbf{t}_{s},
$$

where $k_{T}$ is the tangential stiffness coefficient, $\mathbf{v}_{s}$ is sliding velocity and $\delta_{T}=\int_{t_{0}}^{t} \mathbf{v}_{s}(\tau) \cdot \mathbf{t}_{s} d \tau$ is the tangential displacement. $\eta_{T}$ is the rolling damping coefficient. When the magnitude of the tangential force reaches a critical value $F_{s, c r i t}=\mu\left|F_{n}\right|$, where $\mu$ is the friction coefficient, the surfaces of the two particles start to slide against each other and the tangential force is given by the Amonton friction expression $F_{s}=-F_{s, c r i t}$.

Similarly, the twisting model is given as

$$
M_{t}=-k_{Q} \int_{t_{0}}^{t} \Omega_{T}(\tau) d \tau-\eta_{Q} \Omega_{T}
$$

where $\Omega_{T}=\left(\boldsymbol{\Omega}_{p, i}-\boldsymbol{\Omega}_{p, j}\right) \cdot \mathrm{n}$ is the relative twisting rate, and $k_{Q}$ and $\eta_{Q}$ are the torsional stiffness and the dissipation coefficient, respectively. Similar to the sliding resistance, particles start to 
spin over each other when the magnitude of the imposed twisting moment equals a critical value $M_{t, c r i t}$, which is given as $M_{t, c r i t}=3 \pi a F_{c r i t} / 16$. For twisting moments with magnitude greater than $M_{t, c r i t}$, the torsional resistance is given by $M_{t}=-M_{t, c r i t}$. in the numerical framework. Lubrication force arises from the radial pressure in the interstitial fluid being squeezed from the gap between two close solid surfaces. In the current LBM-DEM, when the gap between the particles and the wall becomes less than the lattice resolution, i.e. one lattice unit, the local viscous flow is not fully resolved. Therefore, an analytical representation of the lubrication force in the local unresolved flow is constructed according to the lubrication theory [48],

$$
\mathbf{F}_{l u b}=-6 \pi \mu_{f} r_{p}^{2} \mathbf{v}_{R}(h)\left(\frac{1}{h}-\frac{1}{h_{c r i t}}\right),
$$

where $\mathbf{F}_{l u b}$ is the lubrication force, $\mathbf{v}_{R}(h)$ is the relative velocity at the gap $h$, and $h_{\text {crit }}$ is critical gap distance to trigger the lubrication correction. The critical distance used in our LBM-DEM framework is set to be one lattice unit, i.e. $h_{c r i t}=1.0 \times \Delta x$. As a result, the total fluid force acting on the particle can be smoothed from hydrodynamic force to the limiting leading-order lubrication force when the particle is approaching the wall, namely,

$$
\mathbf{F}_{f}=\left\{\begin{array}{ll}
\mathbf{F}_{\text {hydro }}, & h \geq h_{\text {crit }} \\
\mathbf{F}_{\text {hydro }}+\mathbf{F}_{\text {lub }}, & h<h_{\text {crit }}
\end{array} .\right.
$$


However, it should be noted that the lubrication correction is not applied in the particle-particle

356

357

358

359 interaction, in order to avoid the so-called "Stokes Paradox", which results in an infinite lubrication force when the gap approaches zero and leads to irrational collisions.

\subsection{Time step scheme in LBM-DEM coupling}

The coupling of the LBM and DEM explicit schemes necessitates matching of their respective critical time steps $[49,50]$. Stability criteria requires the DEM time step to be less than a critical value. However, the LBM the time step implicitly depends on the computational parameters. Both time steps can be varied by a number of orders of magnitude and therefore the ratio between two time steps can be much smaller or much greater than one. Consequently, an efficient technique to handle any disparity in the time steps and to couple the two explicit solution schemes is necessary.

According to Li and Marshall $[44,45]$, the critical time step in DEM must be around the order of the contact time between two colliding particles, which is estimated as

$$
t_{c} \propto R\left[\frac{\rho_{p}^{2}}{E^{2} v_{R}}\right]^{0.2}
$$

This time step is normally in the range of $10^{-6} \sim 10^{-9} \mathrm{~s}$ depending on the particle properties. On the other hand, the time step in the LBM can be inversely calculated from Eq. (5). Hence, in our LBM-DEM coupling scheme a time step ratio $\lambda$ is employed as $\lambda=\Delta t_{L B M} / \Delta t_{D E M}$. The time steps in both LBM and DEM will be estimated according to the simulation setup in order to 

than one, even though in most of our cases it indeed is. As a result, in the simulations where $\Delta t_{L B M}<\Delta t_{D E M}$ the critical time step can be set as the LBM time step. When $\Delta t_{L B M}>\Delta t_{D E M}$ a subcycling approach is taken, which allows the execution of a number of consecutive DEM time steps within a single LBM time step. It is important to note that during DEM sub-cycling the hydrodynamic forces and torques are not updated.

\subsection{Model setup}

384

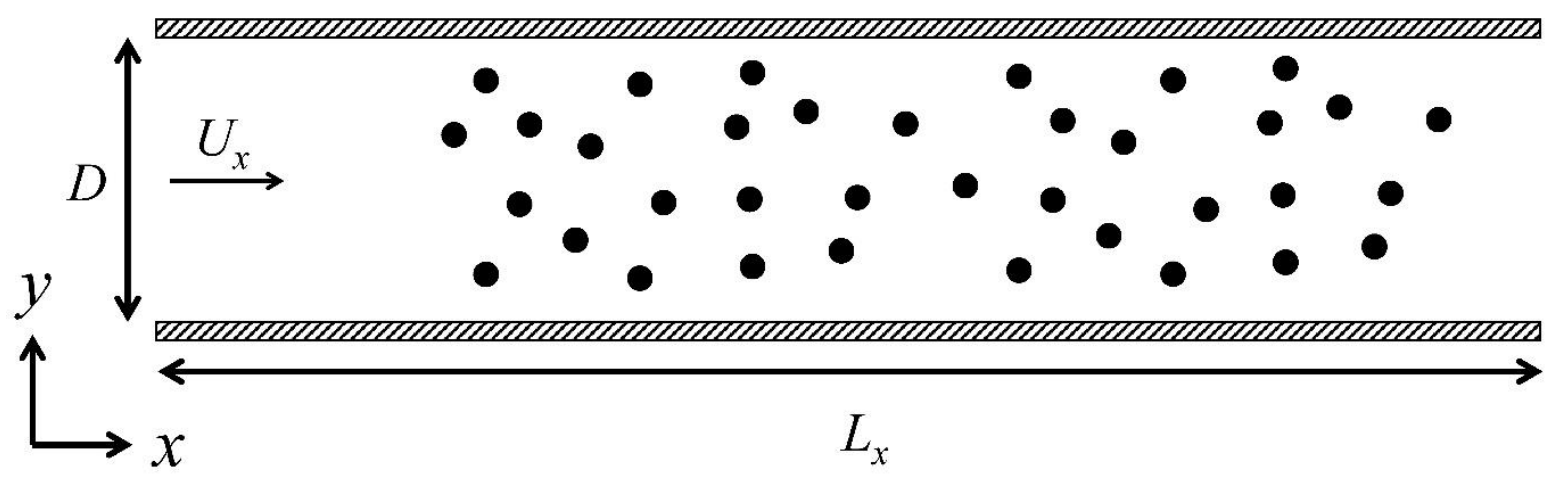

Fig. 4 Schematic of the simulation setup.

Pressure-driven flows of non-Brownian particle suspensions through a two-dimensional channel, as shown in Fig. 4, are considered. The channel is bounded by planar walls at the top and bottom and periodic boundary conditions are applied in the horizontal direction. The suspending fluid is assumed to be Newtonian and the density of the particle is the same as that of the fluid, i.e. the particles are neutrally buoyant. The pressure gradient, which drives the fluid and the particles to flow, is implemented by a constant body force. The initial positions of the particles are randomly distributed in the channel. As the suspension flows over, the 
particles will migrate to their equilibrium positions, forming a steady solid-liquid multiphase flow.

In the LBM computation, the physical parameters are usually dimensionless. Therefore, a unit conversion scheme between the real parameters and the computational ones is established. In the dimensionless lattice scheme, the size of the simulation domain is $L_{x} \times D=500 \times 100$, while the suspended particles are monodisperse with diameter $d=6$ and 12, respectively, which gives a corresponding channel-to-particle size ratio $\alpha=D / d=16.7$ and 8.3. The particle concentration is varied between $1 \%$ to $50 \%$. The pressure gradient ranges between $1.92 \times 10^{-6}$ and $4.8 \times 10^{-5}$, resulting in a channel Reynolds number (with no particles) of $4 \sim 100$ based on the 2D HagenPoiseuille law [51]. It should be noted that with the increase of particle concentration, the channel Reynolds number will decrease. In order to decouple the effect of particle concentration, the Reynolds number of channel with no particles, $R e_{0}$, is denoted as the characteristic Reynolds number, hereinafter. However, it is very difficult to determine $R e_{0}$ for a dense particulate flow without knowing the flow velocity. Therefore, the $R e_{0}$ is tuned by varying the pressure gradient rather than the velocity. Table I summarizes the most important computational parameters, including those used in DEM. Other parameters can be found in the literature [52-53]. Note that the Youngs modulus is reduced by two orders of magnitude compared with the real physical value, in order to use a larger DEM time step so that the simulations are less computationally expensive. 
Table I A list of simulation parameters

\begin{tabular}{lll}
\hline \hline Physical parameters & Actual & Lattice value (dimensionless) \\
\hline Fluid $(\mathrm{LBM})$ & & \\
Channel length $\left(L_{x}\right)$ & $0.02 \mathrm{~m}$ & 500 \\
Channel height $(D)$ & $0.004 \mathrm{~m}$ & 100 \\
Fluid density $\left(\rho_{f}\right)$ & $1,000 \mathrm{~kg} / \mathrm{m}^{3}$ & 1 \\
Fluid kinematic viscosity $\left(v_{f}\right)$ & $1.0 \times 10^{-6} \mathrm{~m}^{2} / \mathrm{s}$ & 0.2 \\
Relaxation parameter $(\tau)$ & & 1.1 \\
Fluid time step $\left(\Delta t_{L B M}\right)$ & $3.2 \times 10^{-4} \mathrm{~s}$ & 1 \\
Time step ratio $(\lambda)$ & 150 & 150 \\
Pressure gradient $\left(\Delta P / L_{x}\right)$ & $0.75 \sim 18.8 \mathrm{~Pa} / \mathrm{m}$ & $1.92 \times 10^{-6} \sim 4.8 \times 10^{-5}$ \\
Channel Reynolds number & $4 \sim 100$ & $4 \sim 100$ \\
with no particles $\left(R e_{0}\right)$ & & \\
\hline Particle $(\mathrm{DEM})$ & & 0.33 \\
Particle diameter $(d)$ & $2.4 \times 10^{-4}, 4.8 \times 10^{-4} \mathrm{~m}$ & 6,12 \\
Particle mass density $\left(\rho_{p}\right)$ & $1,000 \mathrm{~kg} / \mathrm{m}^{3}$ & 1 \\
Particle concentration $(\phi)$ & $1 \% \sim 50 \%$ & 0.3 \\
Youngs modulus $(E)$ & $2.5 \times 10^{8} \mathrm{~Pa}$ & 0.33 \\
Poisson ratio $(v)$ & 0.3 & \\
Friction coefficient $(\mu)$ & & \\
\hline \hline
\end{tabular}

418 3. Migration of dense particle suspensions in a 419 Poiseuille flow

A systematical study on the migration of particle suspensions in a planar Poiseuille flow with different concentrations is performed with the parameters shown in Table I. As mentioned 
above, the flow is driven by the pressure gradient with the specified body force. As the particle concentration increases, obviously the fluid will be accelerated more slowly since the drag resistance caused by the particles increases, which leads to a longer time for the solid-liquid flow to develop into a steady state. Figure 5 shows the snapshots of the flow field as well as the particle positions with the same particle concentration $(\phi=10 \%)$ but different characteristic Reynolds numbers, $R e_{0}$. It can be seen that at a small $R e_{0}=4$, the lateral migration develops very slowly. Only part of the particles migrate laterally at the end of the computation time $t=1 \times 10^{6}$. However, with the increase of $R e_{0}$, a full migration, which means that all the particles migrate laterally to their equilibrium positions, is developed very quickly. For $R e_{0}=40$, it occurs around $t=3 \times 10^{5}$, while for $R e_{0}=100$, it takes place even more earlier around $t=1 \times 10^{5}$. It is also noted that as the particle concentration increases, the flow velocity decreases compared with the pure channel flow. To better to illustrate the effects of particle concentration, Fig. 6 shows the flow field as well as the particle positions with different $\phi$ and $\operatorname{Re}_{0}$. The velocity shown in the contour is normalized with the maximum velocity of the corresponding pure channel flow. It is clear that with a low particle concentration $(\phi=1 \%)$, the velocity profiles still look like parabolic for different $R e_{0}$. However, as $\phi$ increases to $40 \%$, the velocities decrease drastically by approximately an order of magnitude.

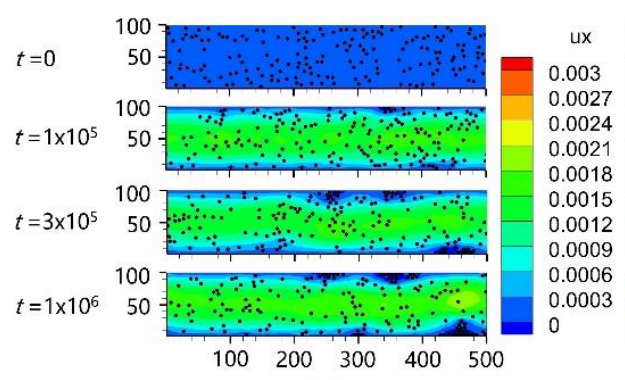

(a)

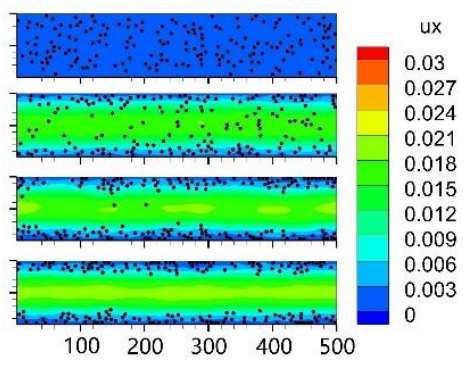

(b)

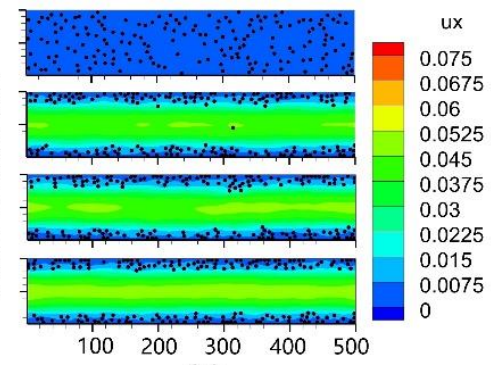

(c) 

but different characteristic Reynolds numbers, (a) $R e_{0}=4$, (b) $R e_{0}=40$, and (c) $R e_{0}=100$. The four subplots from the top to bottom in each column correspond to different time points of

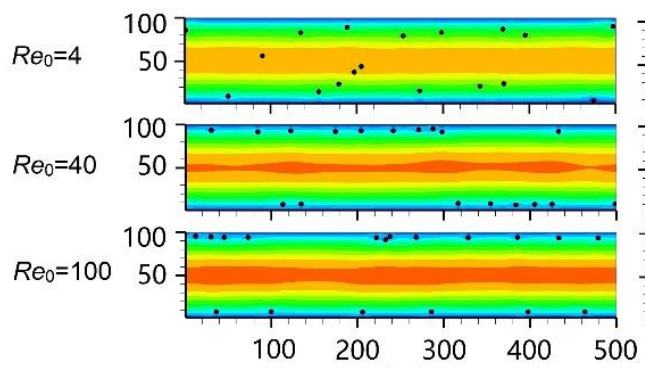

(a)

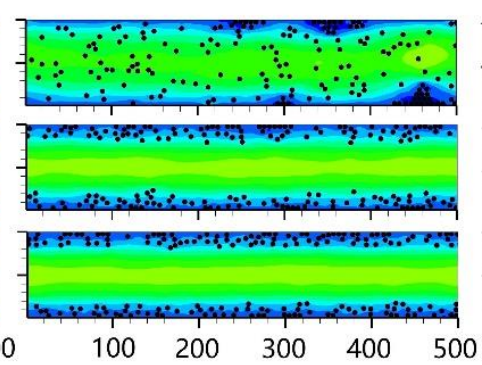

(b)

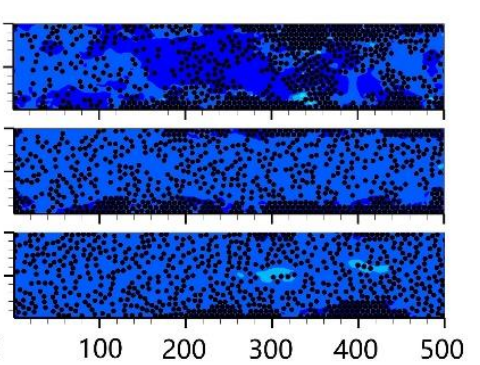

(c)

447 Fig. 6 Snapshots of the flow field and the particle positions $(D / d=16.7)$ with different particle concentrations, (a) $\phi=1 \%$, (b) $\phi=10 \%$, (c) $\phi=40 \%$. The three subplots from the top to bottom in each column correspond to different characteristic Reynolds numbers of $\operatorname{Re}_{0}=4,40$, and 100 , respectively. All the snapshots are taken at the time point of $t=1 \times 10^{6}$. The colormap for each $R e_{0}$ is the same as shown in Fig. 5. details. To make sure that the statistics of the particle positions is reliable, we first check whether the equilibrium state is reached by means of tracking the radial position of all the particles, which is defined as $y_{r}=2 y-D / D$. Figure 7 shows the average radial position $<\left|y_{r}\right|>$ of all the particles as a function of the computation time for a typical case with $\operatorname{Re}_{0}=40$ and

$D / d=16.7$. It can be seen that $<\left|y_{r}\right|>$ for the relatively dilute cases with $\phi \leq 10 \%$ rises rapidly and reaches a plateau at the time $t=4 \times 10^{5}$. On the other hand, for the dense suspensions with 
$\phi=20 \% \sim 50 \%,<\left|y_{r}\right|>$ increases to a stable value only after $t=8 \times 10^{5}$, which takes twice the in all our cases.

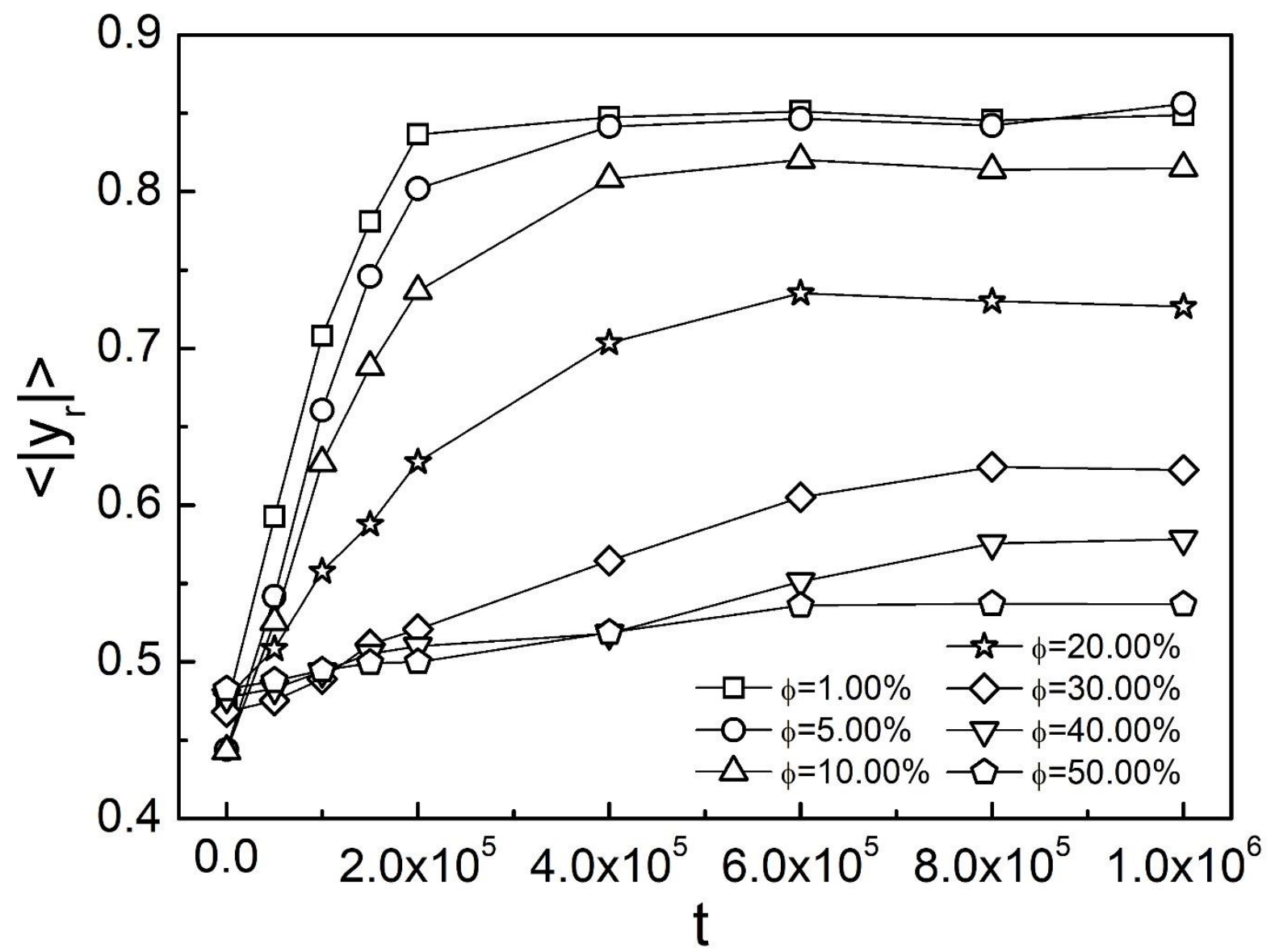

464

Fig. 7 Average radial position of all the particles as a function of the computation time for

$$
R e_{0}=40 \text { and } D / d=16.7 \text {. }
$$

Once the particle suspensions reach the steady state, the local volume fraction along the channel width is measured. Note that the volume in this study is actually the area. For convenience, we the term "volume" is still used. The channel width is first divided into a number of intervals in the $y$-direction. Then the local volume fraction is determined as the ratio 
472 of the total particle volume in each interval to the total volume of this interval. Note that many 473 particles may lie across two neighbouring intervals. In this case only part of the volume is 474 calculated. Figure 8 shows the volume fraction profiles as a function of the radial position for 475 different characteristic Reynolds numbers $R e_{0}$ and size ratios $D / d$. It is found that with a fixed $476 R e_{0}$, two obvious peaks at $\left|y_{r}\right|>0.6$ can be observed at a relatively low concentration $\phi \leq 10 \%$. 477 A large gap, where the local volume fraction is zero, lies between the two peaks, indicating that most of the particles are focused at a certain radial position. At a relatively high concentration $\phi=20 \%-50 \%$, the gap becomes smaller with the increase of the particle concentration. When $\phi \geq 40 \%$, the local volume fraction profile seems to grow a peak at the centerline, implying that particles tend to migration to the center of the channel in the dense case, which is consistent with the previous findings [54-56]. In Poiseuille flows, the shear rate varies across the channel, which results in an inhomogeneous stress field. As a result, the suspended particles tend to migrate into the center region of the channel. In addition, for different $R e_{0}$ and $D / d$, slight differences in the volume fraction profiles are also observed. For instance, for $D / d=16.67$ and $\phi=30 \%-40 \%$, the volume fraction of $R e_{0}=100$ at the centerline is lower than that $R e_{0}=20$ (see Fig. 8a and 8b), which indicates that increasing the channel Reynolds number facilitates the migration of particles. Same observations can be found for $D / d=8.33$. On the other hand, for $R e_{0}=100$ and $\phi=30 \%-40 \%$, the volume fraction of $D / d=16.67$ at the centerline is lower than that of $D / d=8.33$ (see Fig. $8 \mathrm{~b}$ and $8 \mathrm{~d}$ ), which suggests that smaller particles are easier to 491 migrate. 

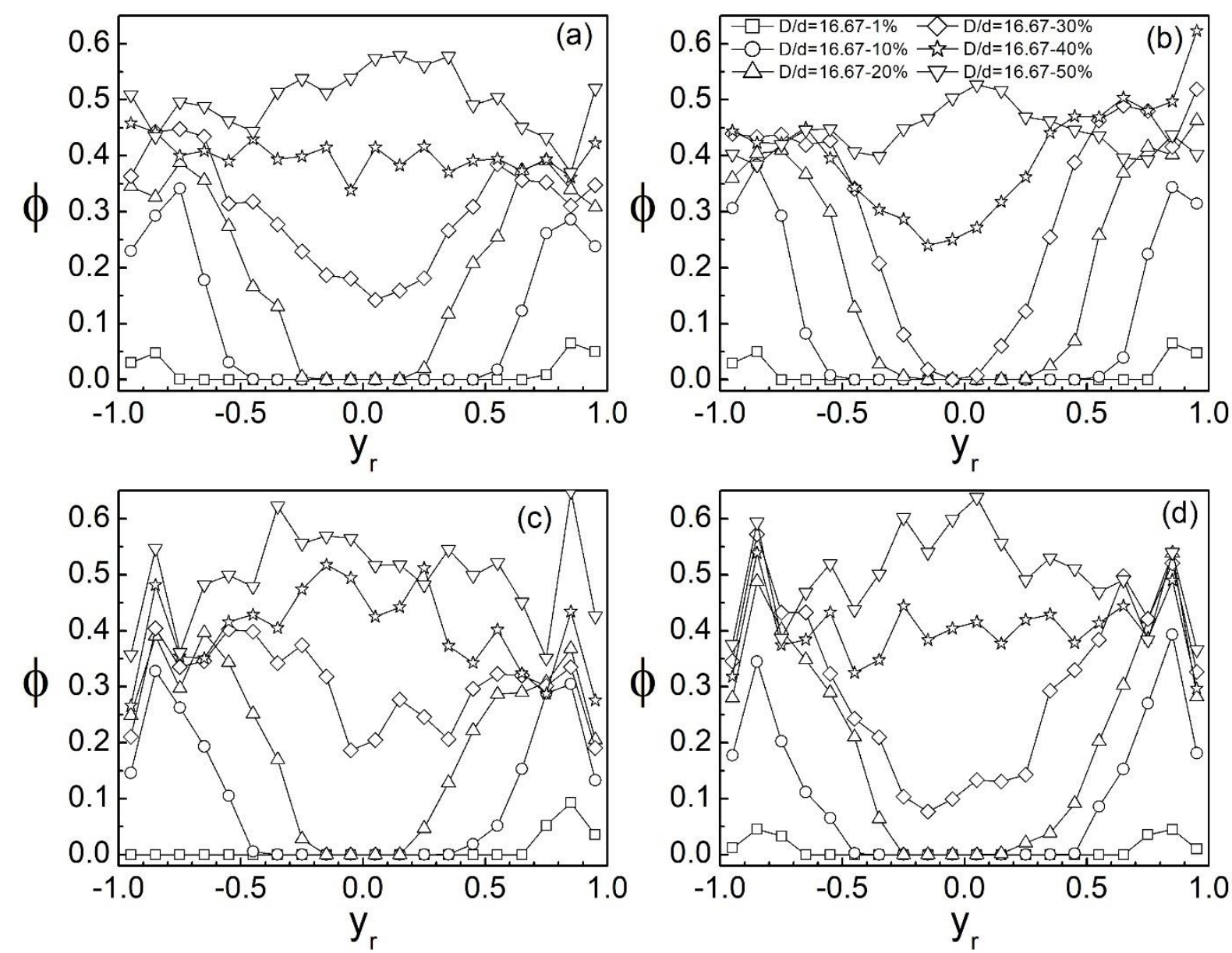

Fig. 8 The volume fraction profiles for (a) $D / d=16.67, R e_{0}=20$, (b) $D / d=16.67, R e_{0}=100$, (c)

$$
D / d=8.33, R e_{0}=20 \text { and (d) } D / d=8.33, R e_{0}=100 \text {. }
$$
impose significant effects on the migration behaviour of the particle suspensions. To quantitatively evaluate their influences, Choi et al.'s work [57] is followed and the degree of inertial migration is defined with the following function,

$$
P_{f}=\sum P D F\left(\left|y_{r}\right| \geq \frac{1}{2} R\right)
$$

Eq. (24) calculates the sum of the probability distribution function (PDF) of the particles in the 
upper and lower quarter of the channel. Considering that the Segré and Silberberg effect gives the radial equilibrium position around 0.6 , if all the particles migrate to their equilibrium positions, then we have $P_{f}=1$. Otherwise if all the particles are uniformly distributed in the channel, Eq. (24) gives $P_{f}=0.5$. Figure 9 shows the degree of inertial migration as a function of particle concentration for various $D / d$ and $R e_{0}$. Generally, $P_{f}$ decreases from 1 to about 0.5 as $\phi$ increases from $1 \%$ to $50 \%$, implying that all the particles completely migrate to their equilibrium positions in dilute suspensions, but almost remain at a uniform distribution in dense suspensions. Besides, the results for different $D / d$ show similar behaviour. However, the migration behaviour strongly depends on $R e_{0}$. For instance, when $R e_{0}=4, P_{f}$ drops dramatically

513 from 1 to 0.5 as $\phi$ is beyond 5\%, while $P_{f}$ stays at 1 and gradually decreases until $\phi \geq 20 \%$ when $\operatorname{Re}_{0}=100$.

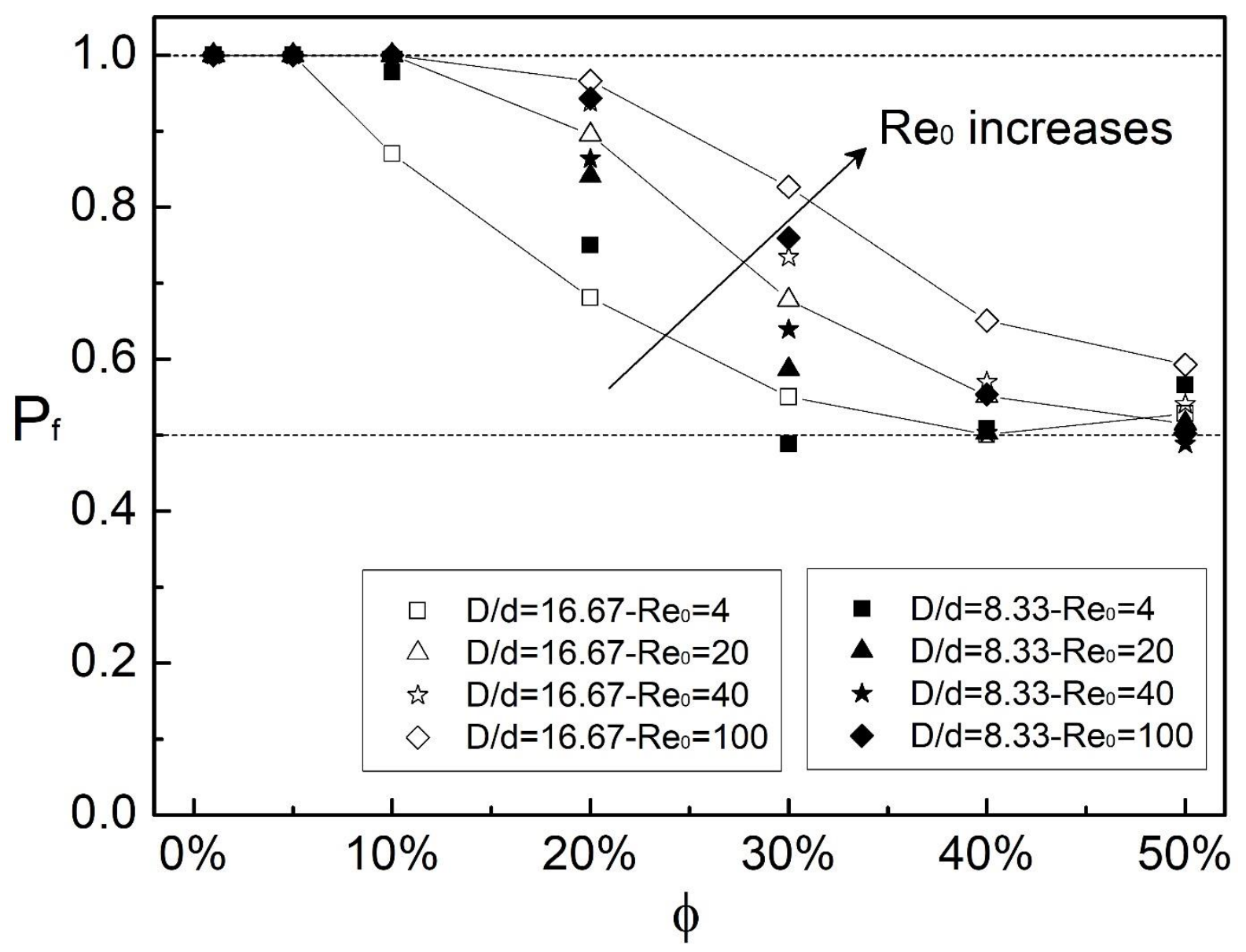


Fig. 9 The degree of inertial migration $P_{f}$ as a function of particle concentration for different

Based on the setup of our system, the potential parameters that may affect the degree of inertial dimensionless parameter, the following general correlation can be yielded based on the dimensional analysis

$$
P_{f} \sim f\left(\operatorname{Re}_{0}, \phi, \frac{D}{d}, \frac{L_{x}}{D}\right) .
$$

If the channel length $L_{x}$ is long enough, $P_{f}$ is expected to be independent of $L_{x} / D$. Furthermore, the particle concentration can be expressed as

$$
\phi=\frac{\pi d^{2} N_{p}}{4 L_{x} D}=\frac{\pi}{4} \frac{d}{D} \lambda,
$$

533 where $\lambda=\frac{N_{p} d}{L_{x}}$ is the length fraction (the number of particle diameters per channel length) 534 [13], the size ratio and channel length can be merged into the particle concentration. Hence, we expect to have a simplified correlation 
539 Generally, the degree of migration increases as the characteristic Reynolds number increases,

540

541 while it decreases when the particle concentration increases.

In previous studies, Zhao et al. [58] proposed a migration index to quantitatively explain the effect of particle concentration on the inertial migration, which is defined as the ratio of the particle-particle interaction force using the Lennard-Jones potential to the inertial lift force with the Asmolov expression [5]. In defining the migration index, the inter-particle distance is replaced by a volume equivalent particle diameter, which depends on the particle concentration and is always greater than the real particle diameter. Thus the Lennard-Jones potential should be attractive and the migration index actually represents the ratio of attractive force to the lift force. For the dense particle flows considered in the present study, the short-range inter-particle collision forces are more important and the attractive force for such particle size is negligible. Therefore, the migration index introduced by Zhao et al. [58] is not applicable here. Alternatively, Di Carlo et al. [13] and Choi et al. [57] proposed two similar approaches to estimate the channel length required for inertial focusing by balancing the inertial lift force and Stokes drag force, and derived a dimensionless focusing number

$$
F_{c}=\operatorname{Re}_{0}\left(\frac{d}{D}\right)^{2} \frac{L_{E}}{D},
$$

where $L_{E}$ is the entry length of the channel. When $F_{c}$ is larger than a critical value, the corresponding degree of inertial migration $P_{f}$ will become 1 , indicating a complete lateral 
migration behaviour. However, this focusing number is not suitable for the cases considered in

560

561

562

563

564

565

566

567 the present study for two reasons: $i$ ) It is derived for dilute suspension, where the flow field is assumed to be not disturbed by the particles. The effect of particle concentration is not fully considered. ii) There is no entry length as periodic boundary conditions are set at the inlet and outlet to explore the flow at the steady state, which is the main focus of this study. In order to find a general criterion to describe the degree of inertial migration when the multiphase flow is fully developed, a new dimensionless focusing number is proposed here to serve as a qualitative indicator on the degree of inertial migration phenomena for dense suspensions.

The new dimensionless focusing number is determined using the simulation results. Through a fitting with two independent variables, the dimensionless focusing number can be expressed as

(1)

$$
F_{c}=\frac{\operatorname{Re}_{0}^{m}}{\phi^{n}}
$$

where $m=0.36$ and $n=2.33$ are obtained from the simulation data presented here. Figure 10 shows the relationship between $P_{f}$ and $F_{c}$, where an empirical fitting is given by

The fitting parameters are $a=0.50, b=0.01$ and $c=6.51$ based on the present simulation data. It can be seen that with the increasing of $F_{c}, P_{f}$ rises from about 0.5 to 1 . Three regimes can be identified based on two critical values of $F_{c}$, which include the upper critical value $F_{c}{ }^{+}$and the lower critical value $F_{c}^{-}$. For the current study, the two critical values are $F_{c}^{+} \approx 300$ and $F_{c}^{-} \approx 30$, 
583 Otherwise, the particles are partially focused in the lateral direction.

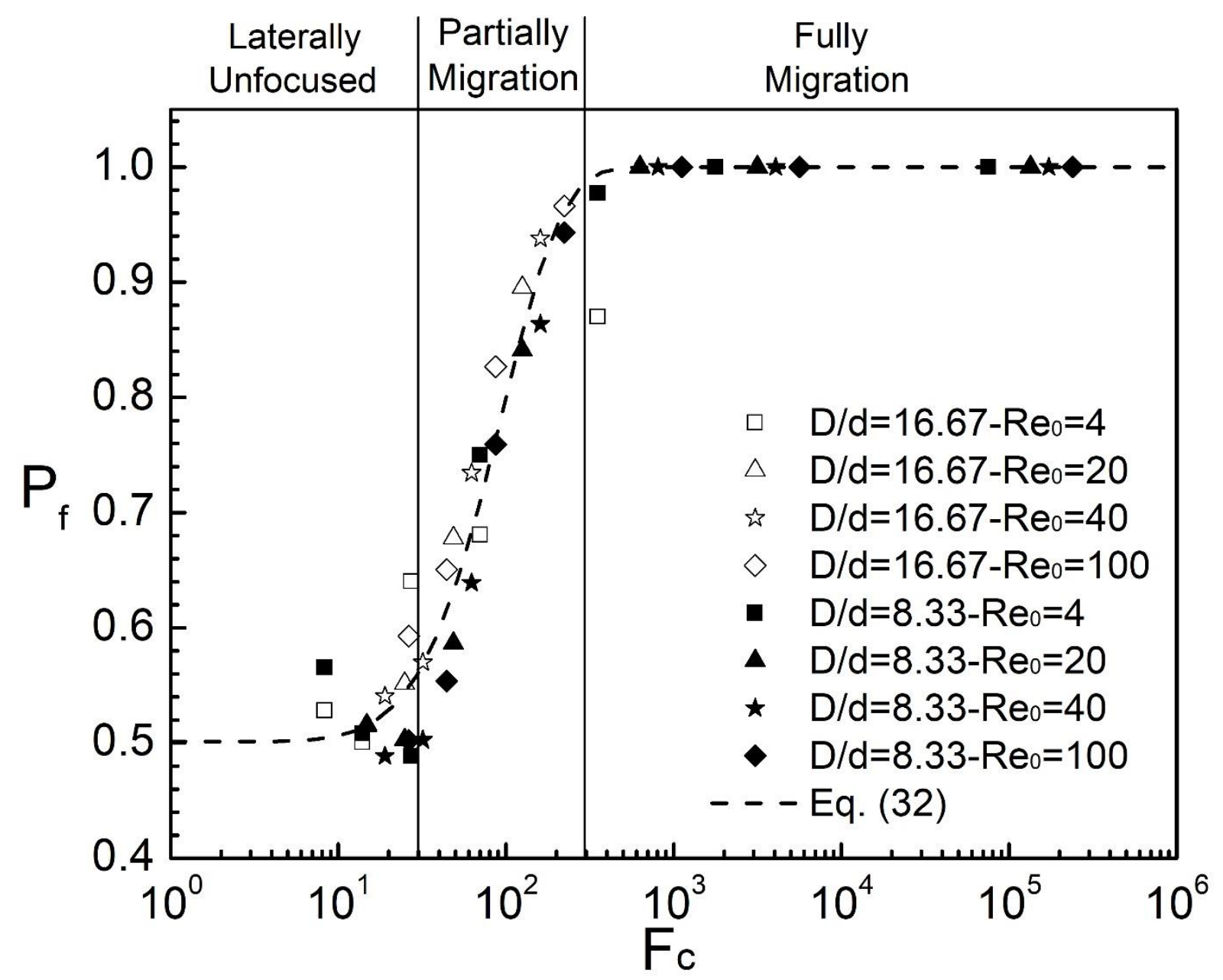

584

Fig. 10 The degree of inertial migration as a function of the focusing number.

Eq. (29) indicates that for a fixed channel, either increasing the characteristic Reynolds number or reducing the particle concentration could enhance the degree of inertial migration. Hence, even though Eqs. (29) and (30) are empirical formulas, they do reflect the underlying physics of inertial migration. Furthermore, more attention must be paid to the assumption that the current study is only performed in 2D. In $3 \mathrm{D}$ cases, the function $P_{f}$ can be defined similarly, 
and the dimensional analysis can be done in the same manner, which yields the same form of the focusing number $F_{c}$. Therefore, similar results are expected and our findings can be extended to 3D cases, except for the different exponents in Eqs. (29)-(30) and critical focusing numbers $F_{c}{ }^{-}$and $F_{c}^{+}$. On the other hand, due to the lack of consideration of the particle-particle lubrication effect, a slightly different migration behaviour may occur, especially in relatively dense suspensions. The presence of inter-particle lubrication force will prevent the particles concentration on the inertial migration are still expected to be similar to those in the cases without consideration of the inter-particle lubrication effect. It is also worth verifying these formulas further, especially experimentally, and exploring the physical background.

\section{Conclusions}

In this paper, a coupled LBM-DEM numerical model is developed, thoroughly validated and then applied to systematically analyse on the effects of particle concentration on the inertial migration of neutrally buoyant particles in a planar Poiseuille flow, especially over a wide range of particle concentration, as previous works on the inertial migration are mainly confined to very dilute suspensions, with very limited studies focusing on the influence of the particle concentration. In this work, we also proposed a general criterion to describe the degree of inertial migration covering a wide range of particle concentration. The numerical results show that the degree of inertial migration decreases with the increasing particle concentration. The 
613

614

615

616

617

618

619

620

621

622

623

624

625

626

627

628

629

630

631

632

influence of particle concentration on the degree of inertial migration depends on the characteristic channel Reynolds number $R e_{0}$, which is defined as the Reynolds number for the corresponding pure channel flow with liquid only and can be determined based on the pressure gradient. For $R e_{0}<20$, evident migration behaviour is only observed with $\phi \leq 5 \%$, while it can still be observed with $\phi \geq 20 \%$ for $\operatorname{Re}_{0}>20$. Finally, a new dimensionless focusing number is proposed to characterise the degree of inertial migration, using which three regimes are identified: the fully migration regime, the partially migration regime and the laterally unfocused regime.

\section{Acknowledgements}

This work is funded by the Engineering and Physical Sciences Research Council (EPSRC, Grants No: EP/N033876/1). W. Liu is grateful to Dr. Duo Zhang and Dr. Nicolin Govender for their helpful suggestions and fruitful discussions in developing the numerical approach.

\section{Appendixes: LBM-DEM model validation}

\section{A1. 2D Poiseuille flow}

The model is first validated for a single phase planar Poiseuille flow in a channel illustrated in Fig. 4. The size of the channel is $10 \times 101$ and the fluid density, kinematic viscosity and relaxation parameter are $1,000 \mathrm{~kg} / \mathrm{m}^{3}, 1 \times 10^{-6} \mathrm{~m}^{2} / \mathrm{s}$ and 1.1 , respectively. The flow is driven by a constant body force, which induces a pressure gradient. By varying the body force, different 

is analytically derived as

$$
U_{x}(y)=\frac{G}{2 \mu_{f}} y(D-y)
$$

where $G$ and $\mu_{f}$ represent the pressure gradient and fluid viscosity, respectively.
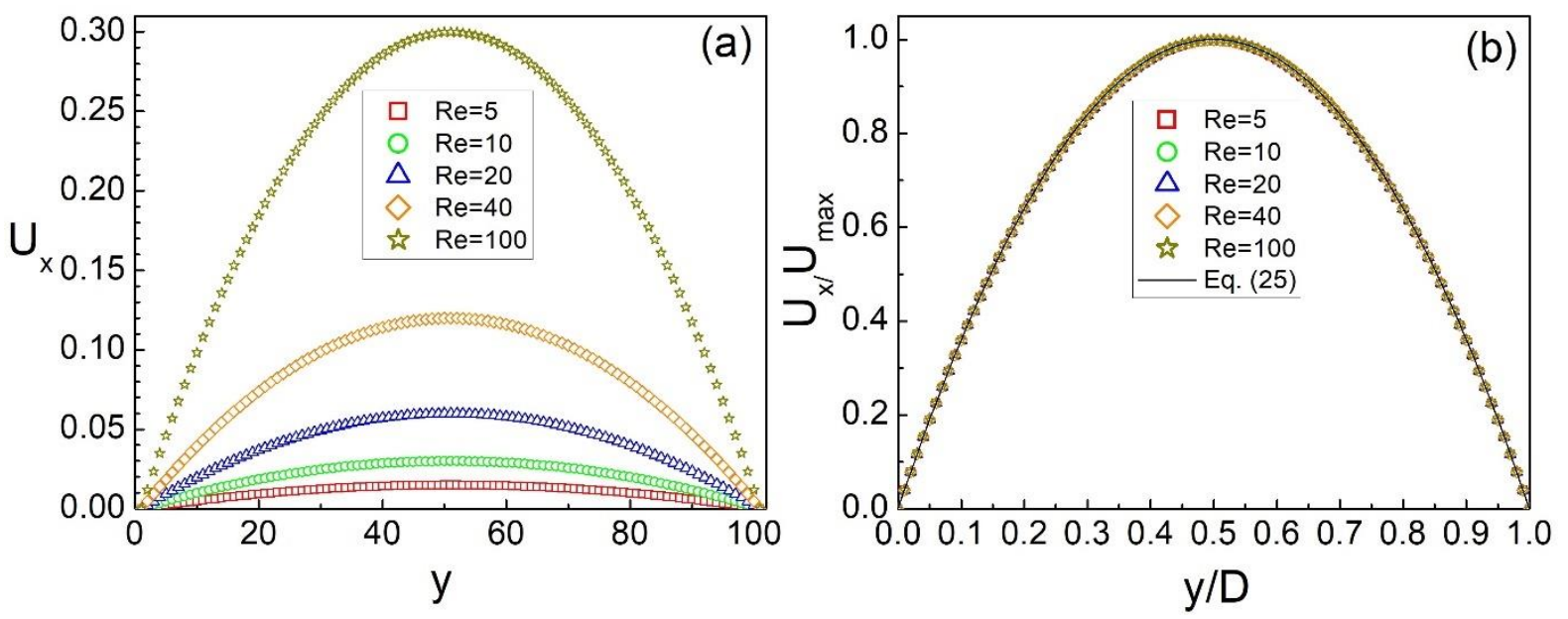

Fig. 11 Velocity profiles for 2D Poiseuille flows. (a) LBM simulation results. (b) The normalized velocity profiles.

Figure 11a shows the velocity profile for Poiseuille flows with different channel Reynolds numbers. The parabolic velocity profiles are well reproduced. Then the velocity is normalized

645 with maximum channel velocity at the centerline, i.e. $U_{\max }=\frac{G D^{2}}{8 \mu_{f}}$, which gives

$$
\frac{U_{x}(y)}{U_{\max }}=4 \frac{y}{D}\left(1-\frac{y}{D}\right) .
$$



be seen that the normalized velocity profiles for various channel Reynolds numbers collapse

651

652

653

654

655

656

657

658

659

660

661

662

663

664

665

666

667

668

669 onto a single curve, which agree perfectly with the theoretical prediction, within a maximum relative error less than $0.2 \%$. Therefore, it is demonstrated that the numerical approach is capable of accurately modelling the single phase fluid flow.

\section{A2. Gravity settling}

In order to validate if the solid-fluid coupling in the developed LBM-DEM can correctly compute the hydrodynamic forces and torques, gravitational settling in $3 \mathrm{D}$ is simulated. A particle is initially placed in the center of cuboid box of size $100 \times 100 \times 100$. The gravity is in the vertical direction and periodic boundary conditions are set in all the faces of the box. The fluid density, kinematic viscosity and relaxation parameter are $1,000 \mathrm{~kg} / \mathrm{m}^{3}, 1 \times 10^{-6} \mathrm{~m}^{2} / \mathrm{s}$ and 0.65 , respectively. The particle density is $3,000 \mathrm{~kg} / \mathrm{m}^{3}$ and three particle radii are considered: $2 \times 10^{-5} \mathrm{~m}, 3.2 \times 10^{-5} \mathrm{~m}$ and $4 \times 10^{-5} \mathrm{~m}$, which equal to 5,8 , and 10 in the lattice unit. The fluid is stationary initially and the particle starts to fall under the gravity. Then a drag force is induced by the fluid to stop the particle from further acceleration, which increases proportionally to the falling velocity. Finally, the drag force will balance the gravitational force and the particle will then fall at a constant speed, which is termed as the terminal velocity. It is well known that with a very low particle Reynolds number $\left(R e_{p}<1\right)$, according to the Stokes law the terminal velocity can be derived analytically as [59]

$$
U_{\text {ter }}=\frac{\left(\rho_{p}-\rho_{f}\right) g d_{p}^{2}}{18 \mu_{f}} .
$$


671

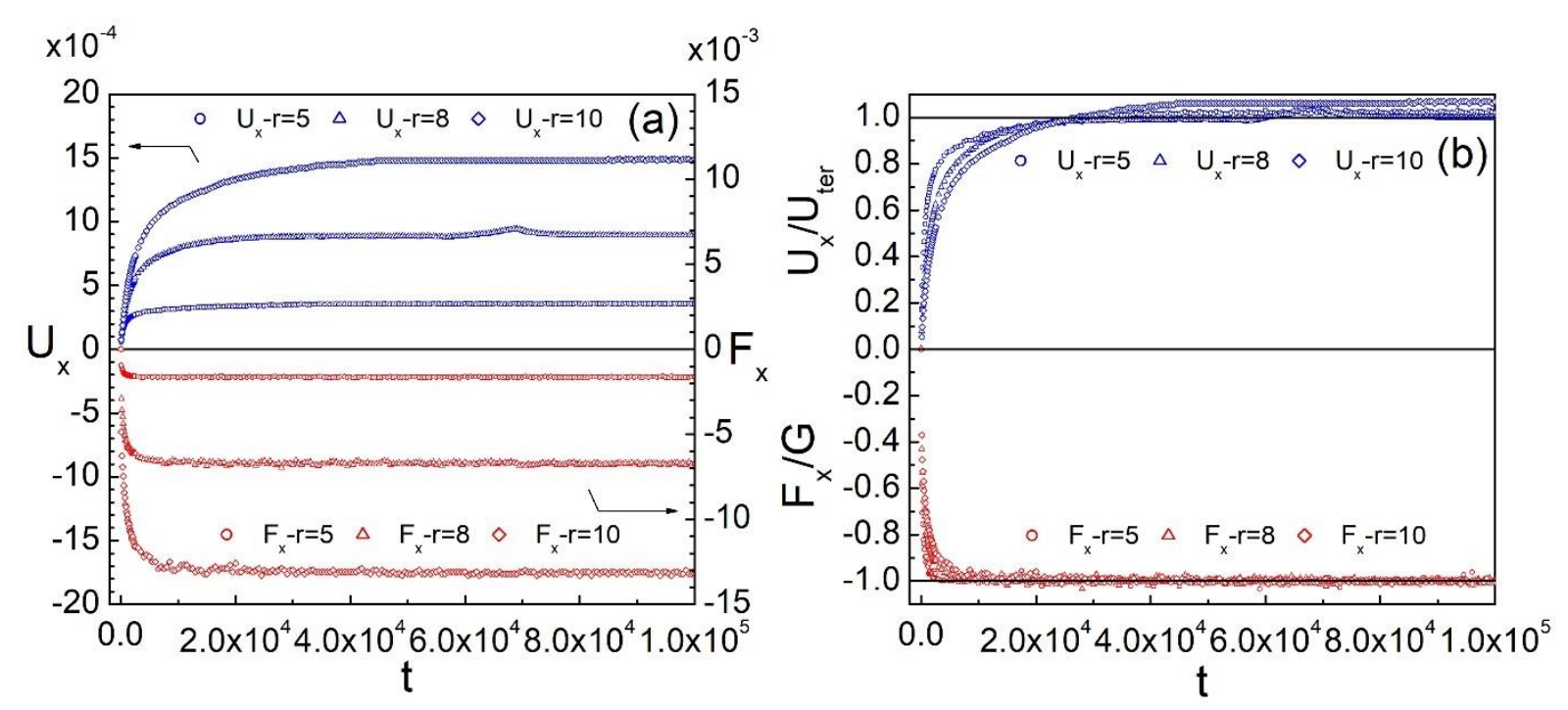

Fig. 12 Velocity and force profiles as a function of the computational time. (a) Simulation results. (b) Normalized velocity and force profiles.

Figure 12 shows the velocity and force profiles as a function of the dimensionless computational time. It can be seen that the particle reaches the steady state very quickly, where the terminal velocity is reached. In all the cases, the drag force equals the effective gravity (gravity minus buoyance). The final terminal velocities are listed in Table II, in which the theoretical predictions (Eq. (A3)) are also given. It should be noted that for the case with particle radius $r=10$, the periodic effect may affect the result as the size of the domain is not large enough compared to the particle size [39], which leads to a relatively large error of $6.5 \%$. However, the relative error for other cases is only within $2.0 \%$, which demonstrates the accuracy of the developed numerical approach. 


\begin{tabular}{ccccc}
\hline \hline Radius & $R e_{p}$ & Simulation & Theory & Relative error \\
\hline 5 & 0.071 & $3.557 \times 10^{-4}$ & $3.488 \times 10^{-4}$ & $2.0 \%$ \\
8 & 0.285 & $8.906 \times 10^{-4}$ & $8.929 \times 10^{-4}$ & $0.3 \%$ \\
10 & 0.594 & $1.486 \times 10^{-3}$ & $1.395 \times 10^{-3}$ & $6.5 \%$ \\
\hline \hline
\end{tabular}

686

Further validations are also performed to verify the lubrication force between the particle and the wall. For this purpose, the experiment of a single particle settling under gravity is reproduced numerically [60]. A particle is initially released from the height of $120 \mathrm{~mm}$ in a box with size of $100 \times 100 \times 160 \mathrm{~mm}$, which corresponds to $50 \times 50 \times 80$ in lattice units. The gravity is

691 in the vertical direction and no-slip wall conditions are set in all the faces of the box. The 692 relaxation parameter is set as 0.05 . The fluid density and viscosity are exactly the same as in the experiment (see Table I in [60]). The particle diameter is $d_{p}=15 \mathrm{~mm}$, which equals to 7.5 in

694 lattice units, and the mass density is fixed at $\rho_{p}=1,120 \mathrm{~kg} / \mathrm{m}^{3}$. Figure 13 shows the particle 695 settling trajectory and velocity profiles as a function of time, which is converted to SI unit to compare with the experimental results. It is clear that, for both the settling trajectory and the velocity, the numerical simulations are in excellent agreement with the experimental results, which confirms the validity and accuracy of our numerical approach. 

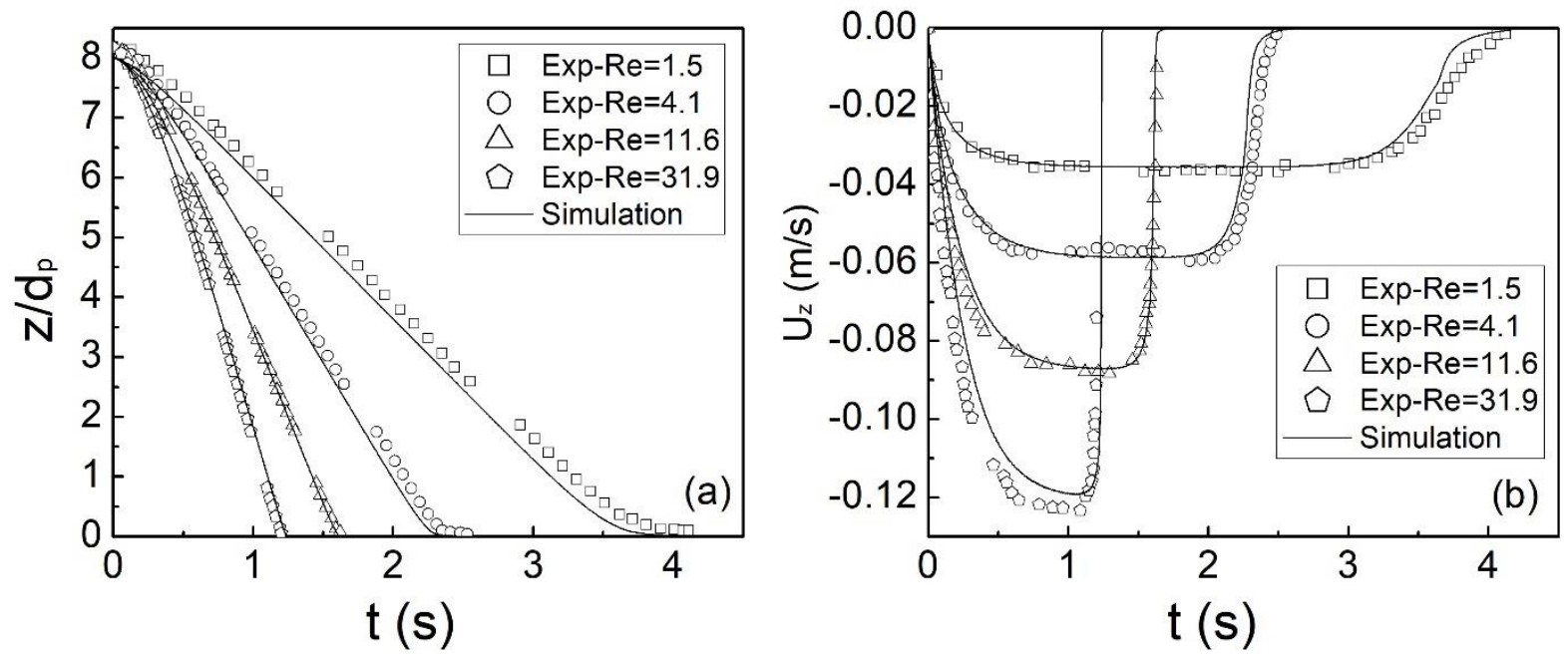

Fig. 13 (a) Particle trajectory and (b) velocity profiles as a function of time.

\section{A3. Inertial migration of a single particle in Poiseuille flows} examine if the model can reproduce the Segré and Silberberg effect. The simulations are performed in a small channel with size of $120 \times 100$ in $2 \mathrm{D}$, with particle diameter of $d=6$ and 12 , which results in two different channel-to-particle size ratios $D / d=16.7$ and 8.3. The flow is driven by the body force. By varying the body force, different channel Reynolds numbers can be achieved. The single particle is initially placed at a random position except for the exact centerline inside the channel. This is because the curvature of the fluid velocity profile at the centerline is zero and the fluid shear becomes zero. Theoretically, if placed on the centerline, the particle will keep moving along it without migrating laterally. Furthermore, the fluid and the particle are both stationary at the beginning. Once the fluid flow starts, the particle starts to move due to the hydrodynamic interactions. After a sufficient long time, the particle will migrate to its lateral equilibrium position and moves at a constant velocity. 

of the dimensionless computation time, where two different series of cases are presented. In

717 Fig. 14a, the channel Reynolds number is fixed at $R e=50$, but the particle is initially placed at 718 different vertical positions of $y_{0}=25 \sim 75$. It can be seen that the particle finally migrates to the 719 lateral equilibrium position around $y / D \approx 0.2$ and 0.8 , which equals to the same radial position 0.6. The further the particle is away from the lateral equilibrium position, the longer time the migration takes as expected. On the other hand, Fig. 14b shows the cases with different $R e$ but the same initial position. Here we choose two symmetric initial positions of $y_{0}=40$ and 60 on each side of the centerline. It is clear that with the increase of the channel Reynolds number, the lateral equilibrium position moves closer to the wall. Moreover, the lateral equilibrium positions are also symmetric about the centerline.
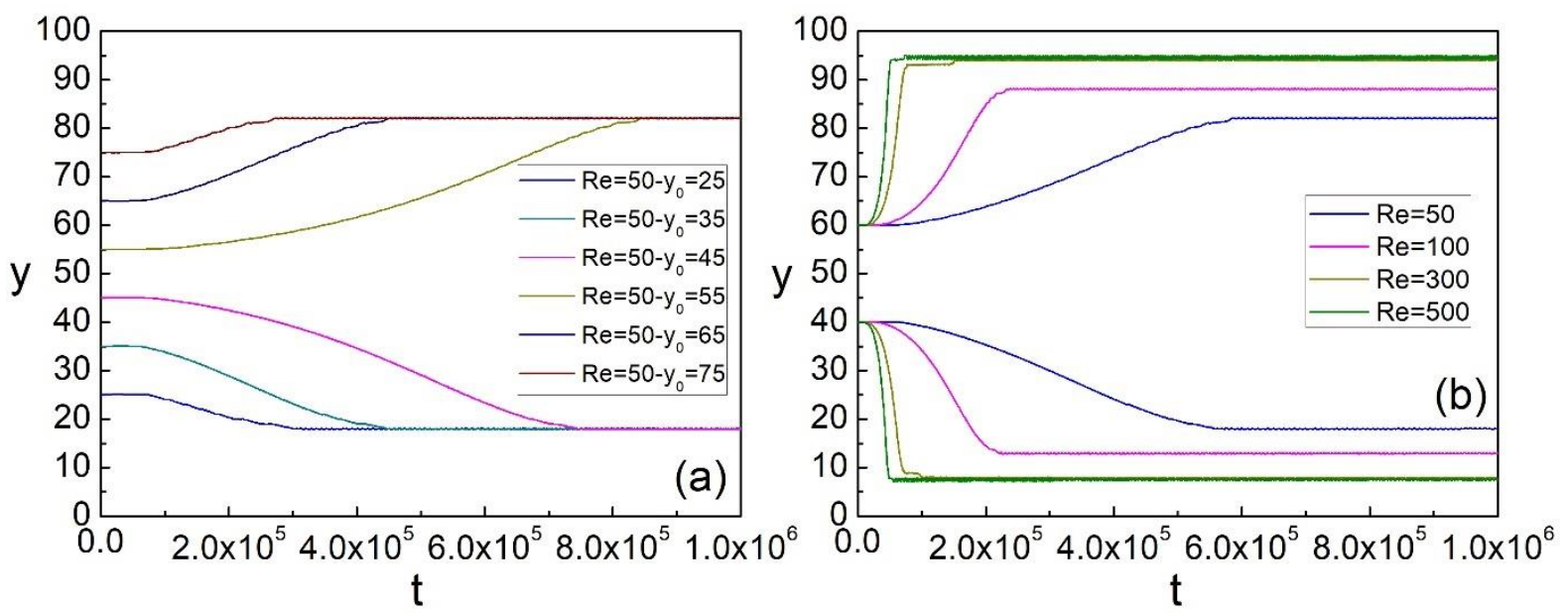

Fig. 14 The migration trace of a single particle $(d=6)$ as a function of the computation time for (a) different initial positions but the same channel Reynolds number $R e=50$, and for (b) the same initial positions but different $R e$. 
732 theoretical ones reported in the literature [2,3,7]. Note that the experiments and theory are in

733 3D. However, as we mentioned in the introduction, the Segré and Silberberg effect is also

734 confirmed to be valid in 2D [1]. Therefore, the present 2D simulation results are comparable

735 and the vertical axis in Fig. 15 is also labelled with radial equilibrium position $r / R$ (equivalent

736 to $|2 y-D| / D$ in 2D) in order to be consistent with the 3D case. From Fig. 15, it can be seen that

737 as the channel Reynolds number increases, the lateral equilibrium position increases from 0.55

738 to 0.88 , i.e. moving towards the wall. With the increase of the channel-to-particle size ratio, the

739 equilibrium positions slightly move up to larger values. The numerical simulations agree well

740 with both the experiment and theory, and hence it can be concluded that the numerical approach

741 can well reproduce the Segré and Silberberg effect.

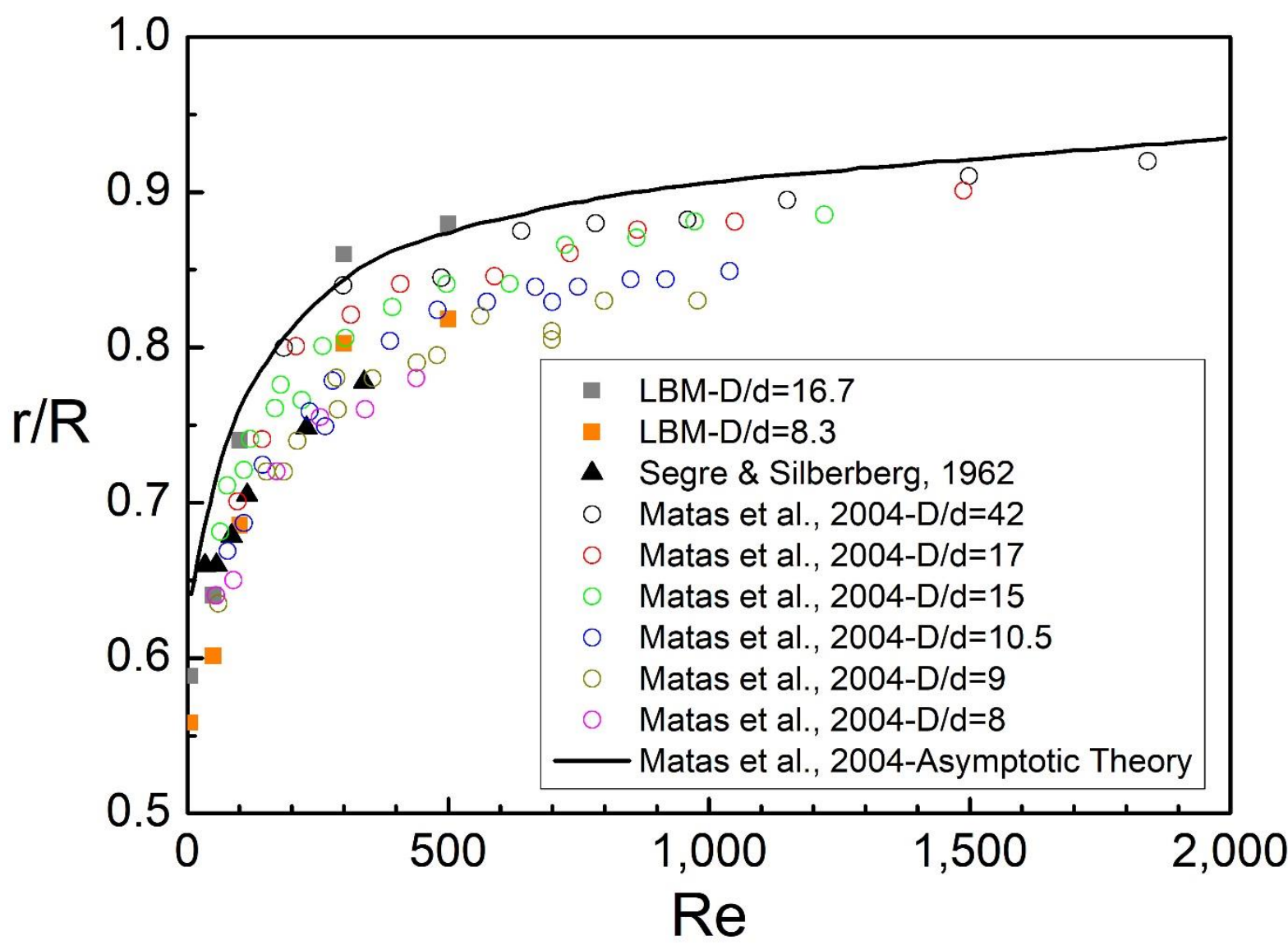

Fig. 15 Lateral equilibrium position of a single particle as a function of the channel Reynolds 


\section{References}

1. Ho B P, Leal L G. Inertial migration of rigid spheres in two-dimensional unidirectional flows. Journal of Fluid Mechanics, 1974, 65(2): 365-400. https://doi.org/10.1017/S0022112074001431

2. Segré G, Silberberg A. Behaviour of macroscopic rigid spheres in Poiseuille flow Part 1. Determination of local concentration by statistical analysis of particle passages through crossed light beams. Journal of Fluid Mechanics, 1962, 14(1): 115-135. https://doi.org/10.1017/S002211206200110X https://doi.org/10.1017/S0022112062001111

4. Schonberg J A, Hinch E J. Inertial migration of a sphere in Poiseuille flow. Journal of Fluid

5. Asmolov E S. The inertial lift on a spherical particle in a plane Poiseuille flow at large channel Reynolds number. Journal of Fluid Mechanics, 1999, 381: 63-87. https://doi.org/10.1017/S0022112098003474

6. Han M, Kim C, Kim M, et al. Particle migration in tube flow of suspensions. Journal of Rheology, 1999, 43(5): 1157-1174. https://doi.org/10.1122/1.551019

7. Matas J P, Morris J F, Guazzelli É. Inertial migration of rigid spherical particles in 
Poiseuille flow. Journal of Fluid Mechanics, 2004, 515: 171-195. https://doi.org/10.1017/S0022112004000254

767

768

769

770

771

772

774

775

776

777

778

779

780

781

782

783

784

785

786

8. Matas J P, Morris J F, Guazzelli E. Lateral forces on a sphere. Oil \& Gas Science and Technology, 2004, 59(1): 59-70. https://doi.org/10.2516/ogst:2004006

9. Matas J P, Glezer V, Guazzelli É, et al. Trains of particles in finite-Reynolds-number pipe flow. Physics of Fluids, 2004, 16(11): 4192-4195. https://doi.org/10.1063/1.1791460

10. Matas, J., Morris, J., \& Guazzelli, É. Lateral force on a rigid sphere in large-inertia laminar pipe flow. Journal of Fluid Mechanics, 2009, 621, 59-67. https://doi.org/10.1017/S0022112008004977

11. Choi Y S, Lee S J. Holographic analysis of three-dimensional inertial migration of spherical particles in micro-scale pipe flow. Microfluidics and Nanofluidics, 2010, 9(4-5): 819-829. https://doi.org/10.1007/s10404-010-0601-8

12. Di Carlo D, Irimia D, Tompkins R G, et al. Continuous inertial focusing, ordering, and separation of particles in microchannels. Proceedings of the National Academy of Sciences, 2007, 104(48): 18892-18897. https://doi.org/10.1073/pnas.0704958104

13. Di Carlo D. Inertial microfluidics. Lab on a Chip, 2009, 9(21): 3038-3046. https://doi.org/10.1039/B912547G

14. Seo K W, Byeon H J, Huh H K, et al. Particle migration and single-line particle focusing in microscale pipe flow of viscoelastic fluids. RSC Advances, 2014, 4(7): 3512-3520. https://doi.org/10.1039/C3RA43522A

15. Seo K W, Kang Y J, Lee S J. Lateral migration and focusing of microspheres in a microchannel flow of viscoelastic fluids. Physics of Fluids, 2014, 26(6): 063301. 
788

789

790

791

792

793

794

795

796

797

798

799

800

801

802

803

804

805

806

807

808

16. Loisel V, Abbas M, Masbernat $\mathrm{O}$, et al. Inertia-driven particle migration and mixing in a wall-bounded laminar suspension flow. Physics of Fluids, 2015, 27(12): 123304. https://doi.org/10.1063/1.4936402

17. Pan Z, Zhang R, Yuan C, et al. Direct measurement of microscale flow structures induced by inertial focusing of single particle and particle trains in a confined microchannel. Physics of Fluids, 2018, 30(10): 102005. https://doi.org/10.1063/1.5048478

18. Feng J, Hu H H, Joseph D D. Direct simulation of initial value problems for the motion of solid bodies in a Newtonian fluid Part 1. Sedimentation. Journal of Fluid Mechanics, 1994, 261: 95-134. https://doi.org/10.1017/S0022112094000285

19. Feng J, Hu H H, Joseph D D. Direct simulation of initial value problems for the motion of solid bodies in a Newtonian fluid. Part 2. Couette and Poiseuille flows. Journal of Fluid Mechanics, 1994, 277: 271-301. https://doi.org/10.1017/S0022112094002764

20. Yang B H, Wang J, Joseph D D, et al. Migration of a sphere in tube flow. Journal of Fluid Mechanics, 2005, 540: 109-131. https://doi.org/10.1017/S0022112005005677

21. Eesa M, Barigou M. Horizontal laminar flow of coarse nearly-neutrally buoyant particles in non-Newtonian conveying fluids: CFD and PEPT experiments compared. International Journal of Multiphase Flow, 2008, 34(11): 997-1007. https://doi.org/10.1016/j.ijmultiphaseflow.2008.06.003

22. Shao X, Yu Z, Sun B. Inertial migration of spherical particles in circular Poiseuille flow at moderately high Reynolds numbers. Physics of Fluids, 2008, 20(10): 103307. https://doi.org/10.1063/1.3005427 
23. Ladd A J C. Numerical simulations of particulate suspensions via a discretized Boltzmann equation. Part 1. Theoretical foundation. Journal of Fluid Mechanics, 1994, 271: 285-309. https://doi.org/10.1017/S0022112094001771

24. Ladd A J C. Numerical simulations of particulate suspensions via a discretized Boltzmann equation. Part 2. Numerical results. Journal of Fluid Mechanics, 1994, 271: 311-339. https://doi.org/10.1017/S0022112094001783

25. Inamuro T, Maeba K, Ogino F. Flow between parallel walls containing the lines of neutrally buoyant circular cylinders. International Journal of Multiphase Flow, 2000, 26(12): 1981-2004. https://doi.org/10.1016/S0301-9322(00)00007-0

26. Chun B, Ladd A J C. Inertial migration of neutrally buoyant particles in a square duct: An investigation of multiple equilibrium positions. Physics of Fluids, 2006, 18(3): 031704. https://doi.org/10.1063/1.2176587

27. Yan Y, Morris J F, Koplik J. Hydrodynamic interaction of two particles in confined linear shear flow at finite Reynolds number. Physics of fluids, 2007, 19(11): 113305. https://doi.org/10.1063/1.2786478

28. Yan Y, Koplik J. Transport and sedimentation of suspended particles in inertial pressuredriven flow. Physics of Fluids, 2009, 21(1): 013301. https://doi.org/10.1063/1.3070919

29. Chun B, Kwon I, Jung H W, et al. Lattice Boltzmann simulation of shear-induced particle migration in plane Couette-Poiseuille flow: Local ordering of suspension. Physics of Fluids, 2017, 29(12): 121605. https://doi.org/10.1063/1.4991428

30. Sun D K, Bo Z. Numerical simulation of hydrodynamic focusing of particles in straight channel flows with the immersed boundary-lattice Boltzmann method. International 
Journal of Heat and Mass Transfer, 2015, 80: 139-149. https://doi.org/10.1016/j.ijheatmasstransfer.2014.08.070

31. Sun D K, Wang Y, Dong A P, et al. A three-dimensional quantitative study on the hydrodynamic focusing of particles with the immersed boundary-Lattice Boltzmann method. International Journal of Heat and Mass Transfer, 2016, 94: 306-315. https://doi.org/10.1016/j.ijheatmasstransfer.2015.11.012

32. $\mathrm{Hu} \mathrm{J}$, Guo Z. A numerical study on the migration of a neutrally buoyant particle in a Poiseuille flow with thermal convection. International Journal of Heat and Mass Transfer, 2017, 108: 2158-2168. https://doi.org/10.1016/j.ijheatmasstransfer.2017.01.077

33. Chen S, Doolen G D. Lattice Boltzmann method for fluid flows. Annual Review of Fluid Mechanics, 1998, 30(1): 329-364. https://doi.org/10.1146/annurev.fluid.30.1.329

34. Bhatnagar P L, Gross E P, Krook M. A model for collision processes in gases. I. Small amplitude processes in charged and neutral one-component systems. Physical Review, 1954, 94(3): 511. https://doi.org/10.1103/PhysRev.94.511

35. Chen H, Chen S, Matthaeus W H. Recovery of the Navier-Stokes equations using a latticegas Boltzmann method. Physical Review A, 1992, 45(8): R5339. https://doi.org/10.1103/PhysRevA.45.R5339

36. Qian Y H, d'Humières D, Lallemand P. Lattice BGK models for Navier-Stokes equation. Europhysics Letters, 1992, 17(6): 479. https://doi.org/10.1209/0295-5075/17/6/001

37. Wolf-Gladrow D A. Lattice-gas cellular automata and lattice Boltzmann models: an introduction. Springer, 2004.

38. Guo Z, Zheng C, Shi B. Discrete lattice effects on the forcing term in the lattice Boltzmann 
$\begin{array}{lllll}\text { method. } & \text { Physical } & \text { Review }\end{array}$

854

855

856

857

858

859

860

861

862

863

864

865

866

867

868

869

870

871

872

873

874

39. Latt J, Chopard B. Lattice Boltzmann method with regularized pre-collision distribution functions. Mathematics and Computers in Simulation, 2006, 72(2-6): 165-168. https://doi.org/10.1016/j.matcom.2006.05.017

40. Zhang R, Shan X, Chen H. Efficient kinetic method for fluid simulation beyond the NavierStokes equation. Physical Review E, 2006, 74(4): 046703. https://doi.org/10.1103/PhysRevE.74.046703

41. Shan X, Yuan X F, Chen H. Kinetic theory representation of hydrodynamics: a way beyond the Navier-Stokes equation. Journal of Fluid Mechanics, 2006, 550: 413-441. https://doi.org/10.1017/S0022112005008153

42. Strack O E, Cook B K. Three-dimensional immersed boundary conditions for moving solids in the lattice-Boltzmann method. International Journal for Numerical Methods in Fluids, 2007, 55(2): 103-125. https://doi.org/10.1002/fld.1437

43. He X, Zou Q, Luo L S, et al. Analytic solutions of simple flows and analysis of nonslip boundary conditions for the lattice Boltzmann BGK model. Journal of Statistical Physics, 1997, 87(1-2): 115-136. https://doi.org/10.1007/BF02181482

44. Li S, Marshall J S, Liu G, et al. Adhesive particulate flow: The discrete-element method and its application in energy and environmental engineering. Progress in Energy and Combustion Science, 2011, 37(6): 633-668. https://doi.org/10.1016/j.pecs.2011.02.001

45. Marshall J S, Li S. Adhesive particle flow. Cambridge University Press, New York, 2014.

46. Bagi K, Kuhn M R. A definition of particle rolling in a granular assembly in terms of 
particle translations and rotations. Journal of Applied Mechanics, 2004, 71(4): 493-501. https://doi.org/10.1115/1.1755693

47. Cundall P A, Strack O D L. A discrete numerical model for granular assemblies. Geotechnique, 1979, 29(1): 47-65. https://doi.org/10.1680/geot.1979.29.1.47

48. Ladd A J C. Sedimentation of homogeneous suspensions of non-Brownian spheres. Physics of Fluids, 1997, 9(3): 491-499. https://doi.org/10.1063/1.869212

881

882

883

884

885

886

887

888

889

890

891

892

893

894

895

896

49. Feng Y T, Han K, Owen D R J. Coupled lattice Boltzmann method and discrete element modelling of particle transport in turbulent fluid flows: Computational issues. International Journal for Numerical Methods in Engineering, 2007, 72(9): 1111-1134. https://doi.org/10.1002/nme.2114

50. Owen D R J, Leonardi C R, Feng Y T. An efficient framework for fluid-structure interaction using the lattice Boltzmann method and immersed moving boundaries. International Journal for Numerical Methods in Engineering, 2011, 87(1-5): 66-95. https://doi.org/10.1002/nme.2985

51. Sutera S P, Skalak R. The history of Poiseuille's law. Annual Review of Fluid Mechanics, 1993, 25(1): 1-20. https://doi.org/10.1146/annurev.fl.25.010193.000245

52. Yang M, Li S, Yao Q. Mechanistic studies of initial deposition of fine adhesive particles on a fiber using discrete-element methods. Powder Technology, 2013, 248: 44-53. https://doi.org/10.1016/j.powtec.2012.12.016

53. Chen S, Li S, Yang M. Sticking/rebound criterion for collisions of small adhesive particles: Effects of impact parameter and particle size. Powder Technology, 2015, 274: 431-440. https://doi.org/10.1016/j.powtec.2015.01.051 
54. Lyon M K, Leal L G. An experimental study of the motion of concentrated suspensions in two-dimensional channel flow. Part 1. Monodisperse systems. Journal of Fluid Mechanics, 1998, 363: 25-56. https://doi.org/10.1017/S0022112098008817

55. Yeo K, Maxey M R. Numerical simulations of concentrated suspensions of monodisperse particles in a Poiseuille flow. Journal of Fluid Mechanics, 2011, 682: 491-518. https://doi.org/10.1017/jfm.2011.241

56. Min K H, Kim C. Simulation of particle migration in free-surface flows. AIChE journal, 2010, 56(10): 2539-2550. https://doi.org/10.1002/aic.12145

57. Choi Y S, Seo K W, Lee S J. Lateral and cross-lateral focusing of spherical particles in a square microchannel. Lab on a Chip, 2011, 11(3): 460-465. https://doi.org/10.1039/C0LC00212G

58. Zhao T, Yao J, Liu K, et al. Investigation of particle inertial migration in high particle concentration suspension flow by multi-electrodes sensing and Eulerian-Lagrangian simulation in a square microchannel. Biomicrofluidics, 2016, 10(2): 024120. https://doi.org/10.1063/1.4946012

59. Lamb H. Hydrodynamics. Cambridge University Press, Cambridge, 1993.

60. Ten Cate A, Nieuwstad C H, Derksen J J, et al. Particle imaging velocimetry experiments and lattice-Boltzmann simulations on a single sphere settling under gravity. Physics of Fluids, 2002, 14(11): 4012-4025. https://doi.org/10.1063/1.1512918 